\title{
DIVERSIDAD Y ENDEMISMO DE LA FAMILIA EUPHORBIACEAE EN MÉXICO
}

\author{
Victor W. SteinManN \\ Instituto de Ecología, A.C. \\ Centro Regional del Bajío \\ Apartado postal 386 \\ 61600 Pátzcuaro, Michoacán
}

\begin{abstract}
RESUMEN
Se calcula que en México las Euphorbiaceae están representadas por 43 géneros y 782 especies, más 32 taxa intraespecíficos: 8 subespecies y 24 variedades. Se propone una lista preliminar de la familia para el país. Los géneros más grandes son Euphorbia (241 spp., 31\%), Croton (124 spp., 16\%), Acalypha (108 spp., 14\%), Jatropha (48 spp., 6\%) y Phyllanthus (41 spp., $5 \%$ ). Juntos representan $72 \%$ de las especies de las Euphorbiaceae conocidas de México. Hay un género endémico del país, Enriquebeltrania y se estima que $57 \%$ de las especies mexicanas son endémicas a su territorio. Empleando el concepto de Megaméxico, este valor aumenta a 78\%. Los taxa introducidos están representados por un género (Ricinus) y 10 especies. Se destacan dos centros de diversidad que corresponden con las vertientes pacífica y atlántica. A nivel de género, las euforbiáceas mexicanas muestran una gran afinidad con las de Centro y Sudamérica; a nivel de especie, en su mayoría son endémicas, pero de las que no lo son, 200 existen también en Centroamérica, 138 en los Estados Unidos, 85 en Sudamérica y 55 en las Antillas. Casi ninguna de las especies mexicanas se encuentra de manera natural en el Viejo Mundo. A pesar de su gran importancia, tanto sistemática como florística, el conocimiento de las Euphorbiaceae en México es aún sumamente pobre.
\end{abstract}

Palabras clave: diversidad, Euphorbiaceae, México.

\begin{abstract}
It is estimated that in Mexico the Euphorbiaceae are represented by 43 genera and 782 species. In addition, there are 32 infraspecific taxa: 8 subspecies and 24 varieties. A preliminary list of the taxa is provided. The most diverse genera are Euphorbia (241 spp., 31\%), Croton (124 spp., 16\%), Acalypha (108 spp., 14\%), Jatropha (48 spp., 6\%), and Phyllanthus (41 spp., 5\%). Together, these represent $72 \%$ of the species present in Mexico. There is one endemic genus, Enriquebeltrania. It is estimated that $57 \%$ of the Mexican species are endemic to the country. Employing the concept of Megamexico, $78 \%$ of the species are endemic. There are ten introduced species and one introduced genus (Ricinus). Within the country, the family is most diverse along the Pacific and Atlantic slopes. At the level of genus, the Mexican Euphorbiaceae show a strong affinity with Central and South America. At the level of species, most Mexican Euphorbiaceae are endemic, but of the species that are not, 200 also occur in Central America, 138 in the United States, 85 in South America, and 55 in the Antilles. Almost no Mexican species are found naturally in the Old World. Despite their great systematic and floristic importance, an adequate understanding of the Mexican Euphorbiaceae is still lacking.
\end{abstract}

Key words: diversity, Euphorbiaceae, Mexico. 


\section{INTRODUCCIÓN}

Con alrededor de 8700 especies ubicadas en 320 géneros (Webster, 1994), la familia Euphorbiaceae es una de las más grandes a nivel mundial y ocupa el sexto lugar en diversidad después de Orchidaceae, Asteraceae, Fabaceae, Poaceae y Rubiaceae. Su distribución es subcosmopolita, y aunque está mejor representada en las regiones tropicales y subtropicales, varios representantes se extienden a las zonas templadas de ambos hemisferios. La familia es sumamente importante, ya que muchos de sus miembros se cultivan para su uso medicinal, industrial, alimenticio y ornamental. Entre las más valiosas están Manihot esculenta Crantz, una de las plantas alimenticias más importantes a nivel mundial, Euphorbia pulcherrima Willd. ex Klotzsch, especie ampliamente cultivada como ornamental y Hevea brasiliensis (Willd. ex Adr. Juss.) Müll. Arg., la fuente principal del hule natural.

Las euforbiáceas son dicotiledóneas, y pertenecen al orden Euphorbiales. La variación morfológica en la familia es enorme, lo que dificulta su caracterización. Sin embargo, la mayoría de las especies se reconocen por sus flores unisexuales, frecuentemente pequeñas, la presencia de un disco floral, un ovario súpero con 3 lóculos, los lóculos con 1 ó 2 óvulos, y frutos típicamente esquizocárpicos capsulares con mericarpos elásticamente dehiscentes. Además, muchas especies tienen látex, hojas con estípulas y varias formas de glándulas.

La familia también es una de las más grandes y diversas de México, y el país constituye el centro de diversidad para muchos géneros. El objetivo principal de este trabajo es presentar un panorama general sobre la familia en este país, con especial énfasis en su estado de conocimiento sistemático, diversidad y endemismo.

\section{FUENTES DE INFORMACIÓN}

Las fuentes de información empleadas en este trabajo fueron principalmente investigaciones de campo, estudios de ejemplares depositados en 21 herbarios de México (ENCB, IEB, MEXU) y del extranjero (A, ARIZ, ASU, BM, CAS, DAV, DS, F, GH, $\mathrm{K}, \mathrm{MICH}, \mathrm{NY}, \mathrm{POM}, \mathrm{RSA}, \mathrm{SD}, \mathrm{TEX}, \mathrm{UC}$, US), así como la revisión de referencias bibliográficas sobre el tema.

\section{CONOCIMIENTO TAXONÓMICO}

Se cuenta con una sola monografía completa de la familia Euphorbiaceae: la obra está publicada en el Prodromus de de Candolle por Boissier (1862, 1866) y Müller (1866). En adición, Gruening (1913), Jablonski (1915), Pax (1910a, 1910b) y Pax y Hoffmann (1911, 1912a, 1912b, 1912c, 1914, 1919a, 1919b, 1919c, 1919d, 1922, 1924) revisaron muchos géneros para Das Pflanzenreich, pero su trabajo es parcial, ya que géneros como Croton, Euphorbia y Phyllanthus, no recibieron un estudio completo. Más recientemente han sido publicadas listas de especies de la tribu Euphorbieae (Oudejans, 1990, 1993) y de toda la familia (Govaerts et al., 2000), además de dos sinopsis de la familia (Webster, 1994; Radcliffe-Smith, 2001). 
Existen pocas revisiones de los géneros mexicanos de Euphorbiaceae, y solamente 16 han sido estudiados en los últimos 60 años (Cuadro 1). Sin embargo, la mayoría de estos trabajos monográficos tratan grupos que no son muy diversos en el país y en total incluyen 122 especies mexicanas. En adición, hay cuatro revisiones de porciones de géneros mexicanos y éstas incluyen 55 especies adicionales, pero ninguna de ellas ha sido publicada formalmente, ya que se trata de tesis de maestría o doctorado. Por lo tanto, 177 especies, o alrededor de 23\% de las especies mexicanas, pertenecen a taxa con una revisión moderna.

Cuadro 1. Taxa mexicanos con una revisión moderna.

\begin{tabular}{|c|c|c|}
\hline Taxon & $\begin{array}{l}\text { Núm. de especies } \\
\text { en México }\end{array}$ & Referencias \\
\hline Argythamnia & 7 & Ingram, 1967; Ingram, 1984 \\
\hline Astrocasia & 4 & $\begin{array}{l}\text { Webster, 1992; Jiménez y Martínez, } \\
2001\end{array}$ \\
\hline Bernardia & 25 & Cervantes, 2002 \\
\hline Chiropetalum & 2 & Ingram, 1980b \\
\hline Cnidoscolus sect. Calyptosolon & 20 & Breckon, 1975 \\
\hline Euphorbia subg. Agaloma sect. & & \\
\hline Alectroctonum & 12 & Ramírez, 1997 \\
\hline Euphorbia subg. Agaloma sect. & & \\
\hline Tithymalopsis & 6 & Huft, 1979 \\
\hline Euphorbia subg. Poinsettia & 17 & Mayfield, 1997 \\
\hline Garcia & 2 & Lundell, 1945 \\
\hline Mabea & 3 & Martínez et al., 2000 \\
\hline Manihot & 20 & Rogers y Appan, 1973; Jiménez, 1990 \\
\hline Margaritaria & 1 & Webster, 1979 \\
\hline Meineckia & 2 & Webster, 1965 \\
\hline Omphalea & 1 & Gillespie, 1988 \\
\hline Pedilanthus & 15 & $\begin{array}{l}\text { Dressler, 1957; Dressler y Sacamano, } \\
\text { 1992; Lomelí y Sahagún, } 1993\end{array}$ \\
\hline Plukenetia & 3 & Gillespie, 1993; Jiménez et al., 2000 \\
\hline Reverchonia & 1 & Webster y Miller, 1963 \\
\hline Stillingia & 12 & Rogers, 1951; McVaugh, 1995 \\
\hline Tetracoccus & 4 & Dressler, 1954 \\
\hline Tragia & 20 & Urtecho, 1996 \\
\hline
\end{tabular}

En varias floras regionales en proceso, por ejemplo la de Veracruz y la del Valle de Tehuacán-Cuicatlán, las Euphorbiaceae no han sido tratadas, pero existen trabajos preliminares para la región de Nueva-Galicia (McVaugh, 1961, 1993, 1995). Además, hay obras importantes para el género Croton en Guerrero (Martínez, 1996a) y Mesoamérica (Martínez, 1996b), un estudio (excluyendo el género Euphorbia) de las especies halófilas y gipsófilas (Cervantes et al., 2001), una sinopsis de la familia en Sonora (Steinmann y Felger, 1997), y una sinopsis de los géneros Phyllanthus y Croton en el occidente tropical 
de México (Webster, 2001) así como trabajos sobre el género Euphorbia en la región del desierto Chihuahuense (Johnston, 1975) y en Baja California (Huft, 1984).

Algunas contribuciones y floras que cubren porciones de los Estados Unidos en su frontera con México también son de utilidad con respecto a las euforbiáceas mexicanas (por ejemplo Webster, 1967; Wheeler, 1941, 1960; Correll y Johnston 1970), al igual que los siguientes estudios de Euphorbiaceae en Centroamérica: Standley y Steyermark (1949), Webster y Huft (1988), Burger y Huft (1995).

\section{DIVERSIDAD Y HABITAT}

Las euforbiáceas contribuyen significativamente a la riqueza de la flora nacional. Tomando como base las referencias bibliográficas y el estudio de ejemplares de herbario, se calcula que en México la familia está representada por 43 géneros y 782 especies (más 32 taxa intraespecíficos: 8 subespecies y 24 variedades), lo que representa más de $3 \%$ de la flora vascular calculada para el país en 22,000 especies (Rzedowski, 1991). Así, también ocupa el sexto lugar en diversidad después de Orchidaceae, Asteraceae, Fabaceae, Poaceae y Cactaceae. En relación al número de Euphorbiaceae a nivel mundial, el país contiene $9 \%$ de las especies y 13\% de los géneros. En el Apéndice 1 se presenta la lista de géneros y especies encontrados en el país.

Existen en México todas las formas de crecimiento representadas en la familia, incluyendo plantas herbáceas anuales (Acalypha pippenii McVaugh y Croton texensis (Klotzsch) Müll. Arg.), hierbas perennes (Acalypha phleoides Cav. y Euphorbia furcillata H.B.K.), geófitas (Euphorbia radians Benth. y Jatropha macrorhiza Benth.), arbustos (Jatropha dioica Cerv. y Tetracoccus fasciculatus (S. Watson) Croizat), árboles (Piranhea mexicana (Standl.) Radcl.-Sm. y Tetrorchidium rotundatum Standl.) y bejucos (Plukenetia carabiasiae J. Jiménez Ram. y Tragia bailloniana Müll. Arg.). La diversidad de formas ecológicas incluye las higrófilas, como Phyllanthus fluitans Benth. ex Müll. Arg., una acuática flotante de Tabasco, y xeromorfas como Euphorbia rossiana Pax, una suculenta endémica del Valle de Tehuacán. En la familia no se conocen epífitas, saprófitas o parásitas.

La familia se encuentra ampliamente distribuida a través de la República desde el nivel de mar hasta más de $3,000 \mathrm{~m}$ de altitud y está representada en los diez principales tipos de vegetación reconocidos por Rzedowski (1978) en México. Sin embargo, alcanza su máxima diversidad y abundancia en los bosques tropicales caducifolios.

Según la clasificación de Webster $(1975,1994)$, la familia se divide en cinco subfamilias, y todas ellas se encuentran representadas en México. En el Cuadro 2 se puede apreciar el número de géneros y especies de cada subfamilia. Las Phyllanthoideae y Oldfieldioideae están poco representadas en comparación con las Acalyphoideae, Crotonoideae y Euphorbioideae.

Aunque aquí se reconocen 43 géneros, este número puede variar un poco, dependiendo del criterio seguido para delimitar algunos de ellos. Además, existe un debate sobre la posición de Drypetes, el que tradicionalmente ha sido tratado dentro de la subfamilia Phyllanthoideae; sin embargo, datos moleculares (Soltis et al., 2000) y embriológicos (Tokuoka y Tobe, 1999) sugieren que Drypetes y algunos géneros 
cercanos no están relacionados con los otros miembros de Euphorbiaceae. Aunque la definición de sus afinidades no ha sido resuelta, aquí se sigue la recomendación de considerar tales géneros dentro de las Putranjivaceae, una familia distinta de Euphorbiaceae, de manera que las tres especies mexicanas de Drypetes están excluidas de este trabajo. Otra diferencia entre los autores es la delimitación de Argythamnia, Chiropetalum y Ditaxis, ya que algunos de ellos (Webster, 1994) los reconocen como géneros independientes, mientras que otros (Ingram, 1980a; McVaugh, 1995) los tratan como un solo género: Argythamnia. Aquí se consideran los tres como distintos. Esser (2001) divide Sebastiania en tres géneros (Sebastiania, Microstachys y Pleradenophora), pero en este trabajo se considera Sebastiania en el sentido amplio. Con respecto a otros grupos, se prefiere reconocer a Euphorbia (vs. Euphorbia, Chamaesyce y Poinsettia) y Croton (vs. Croton, Eremocarpus y Julocroton) en el sentido amplio. A causa de tales diferencias, el número preciso de géneros mexicanos podría variar de 41 a 50 .

Cuadro 2. Representación de las subfamilias en México.

\begin{tabular}{|l|c|c|}
\hline Subfamilia & Géneros & Especies \\
\hline Phyllanthoideae & 8 & 55 \\
Oldfieldioideae & 2 & 5 \\
Acalyphoideae & 16 & 203 \\
Crotonoideae & 7 & 223 \\
Euphorbioideae & 10 & 296 \\
\hline
\end{tabular}

Uno de los aspectos interesantes de la familia es la gran disparidad en el tamaño de los géneros mexicanos. Los más grandes son Euphorbia (241 spp., 31\%), Croton (124 spp., 16\%), Acalypha (108 spp., 14\%), Jatropha (48 spp., 6\%) y Phyllanthus (41 spp., 5\%). Juntos representan $72 \%$ de las especies de Euphorbiaceae en México. A su vez, 27 géneros tienen cinco especies o menos y 11 de ellos están representados por una sola. Es pertinente mencionar que Euphorbia probablemente es el segundo género más grande de la flora de México, después de Salvia.

\section{ASPECTOS FITOGEOGRÁFICOS}

\section{Endemismo}

Se reconoce un género endémico del país, Enriquebeltrania, que es un taxon monotípico distribuido de manera disyunta en la península de Yucatán y en Jalisco. El género Celaenodendron, con anterioridad considerado endémico de México, recientemente ha sido reducido a sinónimo de Piranhea (Radcliffe-Smith y Ratter, 1996), un conjunto neotropical con cuatro especies, de las cuales solamente Piranhea mexicana (Standl.) Radcl.-Sm. \& Ratter (=Celaenodendron mexicanum Standl.) se registra para México. 
Se estima que de las 782 especies existentes en México, 57\% son endémicas al país (Cuadro 3). De acuerdo con Rzedowski (1991), este porcentaje es ligeramente más alto que el nivel de endemismo de todas las plantas del grupo de las fanerógamas de México. Empleando el concepto de Megaméxico ${ }^{1}$ acuñado por Rzedowski (op. cit.), 78\% de las especies son endémicas. Una vez más, esta cifra es un poco más alta que el nivel correspondiente para todas las fanerógamas mexicanas.

Cuadro 3. Especies endémicas de Euphorbiaceae en México.

\begin{tabular}{|l|c|c|}
\hline & Número de especies & Porcentaje \\
\hline México & 445 & 57 \\
Megaméxico 1 & 519 & 67 \\
Megaméxico 2 & 525 & 68 \\
Megaméxico 3 & 613 & 78 \\
\hline
\end{tabular}

Destacan 12 géneros con muchas especies endémicas (Cuadro 4), que en su conjunto alcanzan un nivel de endemismo de $72 \%$, igual al de las cactáceas (Rzedowski, op. cit.). Argythamnia es de interés particular, ya que todas sus especies mexicanas son endémicas del país. Además, cinco de ellas se conocen solamente de un estado y tres de una sola colecta.

Cuadro 4. Géneros con un elevado número de especies endémicas.

\begin{tabular}{|l|c|c|}
\hline Género & $\begin{array}{c}\text { Número de especies endémicas / } \\
\text { Número de especies en total }\end{array}$ & Porcentaje correspondiente \\
\hline Argythamnia & $7 / 7$ & 100 \\
Chiropetalum & $2 / 2$ & 100 \\
Enriquebeltrania & $1 / 1$ & 100 \\
Piranhea & $1 / 1$ & 100 \\
Jatropha & $39 / 48$ & 81 \\
Pedilanthus & $12 / 15$ & 80 \\
Cnidoscolus & $19 / 24$ & 79 \\
Astrocasia & $3 / 4$ & 75 \\
Manihot & $14 / 20$ & 70 \\
Bernardia & $17 / 25$ & 68 \\
Dalembertia & $2 / 3$ & 67 \\
Acalypha & $70 / 108$ & 65 \\
\hline
\end{tabular}

${ }^{1}$ El concepto de Megaméxico es una delimitación de México basada más en fronteras biológicas en lugar de líneas políticas. Megaméxico 1 se refiere a México en adición a las partes de las zonas áridas sonorense, chihuahuense y tamaulipeca en los Estados Unidos. Megaméxico 2 incluye a México y Centroamérica hasta el norte de Nicaragua. Megaméxico 3 a México y ambas extensiones. 
Géneros con centros de diversidad en México

En total, 14 géneros concentran su diversificación en el territorio del país (Cuadro 5), sin contar a Enriquebeltrania y Reverchonia, que son monotípicos. Para algunos de ellos, se trata de un centro primario (Acalypha, Astrocasia, Adelia, Dalembertia, Euphorbia, Garcia, Pedilanthus, Stillingia y Tetracoccus), mientras que para otros es más bien secundario (Bernardia, Cnidoscolus, Croton, Jatropha y Manihot).

Cuadro 5. Géneros con centros de diversificación en México.

\begin{tabular}{|l|c|c|}
\hline Género & $\begin{array}{c}\text { Número de especies en México / } \\
\text { Número de especies en total }\end{array}$ & Porcentaje correspondiente \\
\hline Pedilanthus & $15 / 15$ & 100 \\
Dalembertia & $3 / 3$ & 100 \\
Garcia & $2 / 2$ & 100 \\
Tetracoccus & $4 / 5$ & 80 \\
Astrocasia & $4 / 6$ & 67 \\
Adelia & $6 / 13$ & 46 \\
Stillingia & $12 / 29$ & 41 \\
Bernardia & $25 / 70$ & 36 \\
Cnidoscolus & $24 / 67$ & 36 \\
Jatropha & $48 / 188$ & 25 \\
Acalypha & $108 / 465$ & 23 \\
Manihot & $20 / 108$ & 19 \\
Euphorbia & $241 / 1840$ & 13 \\
Croton & $124 / 1225$ & 10 \\
\hline
\end{tabular}

Especies mexicanas con uso económico

De las euforbiáceas nativas de México, la especie más sobresaliente es Euphorbia pulcherrima, la famosa "noche buena". La planta es propia del bosque tropical caducifolio de la vertiente pacífica, desde Sinaloa a Guatemala; hoy se propaga como ornamental a través del mundo. A nivel de menor importancia, E. fulgens Karw. ex Klotzsch, E. leucocephala Lotsy y E. pteroneura A. Berger también se cultivan. Euphorbia antisyphilitica Zucc., la "candelilla", es una fuente importante de cera vegetal, y en la región desértica Chihuahuense las plantas se cosechan para obtener esta materia prima. Localmente en la vertiente pacífica, especialmente en Sinaloa y Sonora, de especies de Croton sect. Eluteria (por ejemplo, C. niveus Jacq. y C. psuedoniveus Lundell) se aprovechan los tallos para su uso como estacas en los campos agrícolas.

Además de las especies ya mencionadas, ninguna otra es de gran trascendencia económica en la actualidad. Sin embargo, tradicionalmente muchas tienen uso medicinal y posiblemente serán de importancia algún día. Aunque las euforbiáceas tienen reputación de ser plantas venenosas, cabe mencionar que dos especies han sido 
registradas como comestibles en ejemplares del herbario: las hojas de Euphorbia delicatula Boiss., que se usan como especia, y los tubérculos de Euphorbia macropus (Klotzsch \& Garcke) Boiss., que se mastican como chicle.

Especies cultivadas introducidas

Muchas euforbiáceas se cultivan en México por varias razones, pero principalmente como ornamentales. De estas últimas las más importantes son Acalypha hispida Burm., Breynia disticha J. R. \& G. Forst., Codiaeum variegatum (L.) Adr. Juss., Jatropha multifida L., y varios representantes suculentos de Euphorbia como E. tirucalli L. y E. neriifolia L. Aunque no con gran profusión en México, Manihot esculenta, la "yuca", se cultiva a través de los trópicos y es una de las plantas alimenticias más importantes a nivel mundial. Plantaciones de Hevea brasiliensis se observan esporádicamente en la región de los bosques tropicales del sur del país como una fuente de hule natural. Se siembra ampliamente a Ricinus communis como ornamental y de las semillas se extrae el llamado "aceite de ricino" o "aceite de castor", que tiene muchos usos tanto medicinales como industriales. Esta última especie también es adventicia en el país (véase más adelante).

Especies silvestres introducidas

Los taxa introducidos no constituyen un grupo muy importante dentro de las euforbiáceas mexicanas. Están representados por un género (Ricinus) y 10 especies (Acalypha indica L., A. infesta Poepp. \& Endl., Bernardia sidoides (Klotzsch) Müll. Arg., Euphorbia hirsuta L., E. lathyris L., E. peplus L., E. terracina L., Ricinus communis L., Phyllanthus acidus (L.) Skeels, y P. tenellus Roxb.). De estos elementos, solamente dos son comunes y los restantes tienen una distribución restringida. Ricinus communis, originaria del noreste de África tropical, es una de las plantas más frecuentes en México, y se encuentra bien representada a lo largo del país. La otra especie introducida común es Euphorbia peplus, planta de Eurasia que frecuentemente se encuentra alrededor de las casas. Ambas por lo general están confinadas a sitios perturbados, y ninguna es muy agresiva.

Diversidad y endemismo a nivel estatal

En este momento es difícil hacer una evaluación precisa de la diversidad y endemismo de la familia a nivel estatal. Sin embargo, tomando como base las revisiones disponibles que incluyen las 177 especies mencionadas antes y algunos datos subsecuentes (por ejemplo, especies descritas posteriormente y extensiones de distribución), podemos estimar la diversidad y endemismo relativo por cada estado (Cuadro 6). Se destacan dos centros de diversidad que corresponden con las dos vertientes de México. Esto ya se esperaba, porque en estas regiones el bosque tropical caducifolio está mejor desarrollado, y es en este tipo de vegetación donde las euforbiáceas prosperan más. La familia es relativamente menos diversa en el centro del país y también en la península de Yucatán. Con respecto al endemismo, el estado con el mayor número de elementos de distribución restringida es Oaxaca. Sin embargo, la proporción de especies endémicas con respecto al número total es más alta en Baja California Sur. 
Cuadro 6. Diversidad y endemismo a nivel estatal basado en las 177 especies que pertenecen a géneros con una revisión moderna.

\begin{tabular}{|l|c|c|}
\hline Estado & Número total de especies & Número de especies endémicas \\
\hline Jalisco & 48 & 5 \\
Oaxaca & 48 & 7 \\
Chiapas & 45 & 1 \\
Michoacán & 34 & 4 \\
Guerrero & 33 & 6 \\
Sinaloa & 32 & 1 \\
Veracruz & 31 & 3 \\
Tamaulipas & 31 & 2 \\
Nayarit & 29 & 4 \\
Puebla & 29 & 1 \\
Sonora & 29 & 1 \\
San Luis Potosí & 28 & 1 \\
Chihuahua & 26 & 0 \\
Edo. de México & 23 & 2 \\
Durango & 21 & 3 \\
Coahuila & 20 & 0 \\
Hidalgo & 18 & 0 \\
Querétaro & 18 & 0 \\
Colima & 17 & 0 \\
Yucatán & 17 & 2 \\
Nuevo León & 16 & 0 \\
Guanajuato & 14 & 0 \\
Quintana Roo & 13 & 0 \\
Baja California Sur & 12 & 4 \\
Campeche & 12 & 0 \\
Morelos & 12 & 0 \\
Baja California & 10 & 0 \\
Tabasco & 10 & 1 \\
Zacatecas & 8 & 0 \\
Aguascalientes & 2 & 0 \\
Tlaxcala & & \\
\hline
\end{tabular}

\section{Relaciones florísticas}

A nivel de género, las euforbiáceas mexicanas muestran una gran afinidad con las de Centro y Sudamérica. De los 42 géneros nativos en México, 35 también existen en Centroamérica y 36 en Sudamérica, en comparación con las Antillas, en donde también se registran 27 géneros mexicanos, y el Viejo Mundo donde hay 18. Solamente 14 géneros mexicanos se encuentran en los Estados Unidos, pero esta cifra representa toda la diversidad de ese país.

A nivel de especie, la flora mexicana de las euforbiáceas es muy especializada y, como ya ha sido mencionado, en su mayoría son endémicas. De las que no son endémicas, 200 también existen en Centroamérica, 138 en los Estados Unidos, 85 en 
Sudamérica y 55 en las Antillas. Casi ninguna de las especies mexicanas se encuentra en forma natural en el Viejo Mundo. Cabe mencionar que la mayoría de las Euphorbiaceae de los Estados Unidos, y especialmente las encontradas cerca de la frontera con México, son extensiones de taxa mexicanos y principalmente son elementos de zonas áridas o semiáridas. Casi todas las restantes son endémicas de ese país.

\section{CONCLUSIONES}

La familia Euphorbiaceae es una de las más grandes en México, pues cuenta con 782 especies conocidas, ubicadas en 43 géneros. La mayoría de las especies son endémicas y el país representa un centro de diversidad para muchos géneros. A pesar de su gran importancia tanto sistemática como florística, el conocimiento de las Euphorbiaceae en México es aún sumamente pobre; considerando que hasta el momento menos de $25 \%$ de las especies han sido sometidas a una revisión, todavía falta muchísimo para tener un entendimiento básico acerca de la distribución y rareza de la mayoría de los componentes de la familia. No existen esfuerzos conservacionistas de los taxa que sabemos que son raros y se carece de datos acerca de fitogeografía, anatomía, biología de la polinización y números cromosómicos. Por todo lo anterior, lo más importante en este momento es realizar más trabajos monográficos, en particular de los géneros grandes, ya que tales contribuciones son la base para los estudios de conservación.

\section{AGRADECIMIENTOS}

Agradezco a Michael Huft, Grady Webster y Sergio Zamudio por la revisión crítica del manuscrito y a Angélica Cervantes por compartir sus datos sobre Bernardia. El trabajo fue realizado con apoyo económico de una Beca Fulbright-García Robles y del Instituto de Ecología, A.C. (cuenta 902-03).

\section{LITERATURA CITADA}

Boissier, E. 1862. Euphorbieae. In: de Candolle, Prodr. 15(2): 3-188.

Boissier, E. 1866. Euphorbieae (addenda y corrigenda). In: de Candolle, Prodr. 15(2): 1261-1269.

Breckon, G. J. 1975. Cnidoscolus section Calyptosolen (Euphorbiaceae) in Mexico and Central America. Tesis de doctorado. University of California. Davis. $463 \mathrm{pp}$.

Burger, W. y M. Huft. 1995. Euphorbiaceae. W. Burger (ed.). Flora Costaricensis Núm. 113. Fieldiana, Bot. 36: 1-169.

Cervantes M., A. 2002. El género Bernardia (Euphorbiaceae) en México. Tesis de maestría. Universidad Nacional Autónoma de México. México, D.F. 189 pp.

Cervantes M., A., H. Flores O. y J. Valdés. 2001. Las Euphorbiaceae halófilas y gipsófilas de México, excepto Euphorbia. An. Inst. Biol. Univ. Nac. Aut. Méx., Ser. Bot. 72: 1-83.

Correll, D. S. y M. C. Johnston. 1970. Manual of the vascular plants of Texas. Texas Research Foundation. Renner. 1881 pp.

Dressler, R. L. 1954. The genus Tetracoccus (Euphorbiaceae). Rhodora 56: 45-61. 
Dressler, R. L. 1957. The genus Pedilanthus (Euphorbiaceae). Contr. Gray Herb. 182: 1-188.

Dressler, R. L. y C. M. Sacamano. 1992. Pedilanthus connatus (Euphorbiaceae), especie nueva y llamativa de Jalisco. Acta Bot. Mex. 18: 21-24.

Esser, H.-J. 2001. Tribe Hippomaneae. In: Radcliffe-Smith, A. Genera Euphorbiacearum. Royal Botanical Gardens. Kew, Surrey. pp. 352-393.

Gillespie, L. J. 1988. A revision and phylogenetic analysis of Omphalea (Euphorbiaceae). Tesis de doctorado. University of California. Davis. $297 \mathrm{pp}$.

Gillespie, L. J. 1993. A synopsis of neotropical Plukenetia (Euphorbiaceae) including two new species. Syst. Bot. 18: 575-592.

Govaerts, R., D. G. Frodin y A. Radcliffe-Smith. 2000. World checklist and bibliography of the Euphorbiaceae (with Pandaceae). World Checklists and Bibliographies 4. Royal Botanic Gardens. Kew. 1621 pp.

Gruening, G. 1913. Euphorbiaceae-Porantheroideae-Ricinocarpoideae. In: Engler, A. (ed.). Das Pflanzenreich IV. 147. (Heft 58): 1-97.

Huft, M. J. 1979. A monograph of Euphorbia section Tithymalopsis. Tesis de doctorado. University of Michigan. Ann Arbor. 286 pp.

Huft, M. J. 1984. A review of Euphorbia (Euphorbiaceae) in Baja California. Ann. Missouri Bot. Gard. 71: $1021-1027$.

Ingram, J. 1967. A revisional study of Argythamnia subgenus Argythamnia (Euphorbiaceae). Gentes Herb. 10: 1-38.

Ingram, J. 1980a. The generic limits of Argythamnia (Euphorbiaceae) defined. Gentes Herb. 11: 427436.

Ingram, J. 1980b. A revision of Argythamnia subgenus Chiropetalum (Euphorbiaceae). Gentes Herb. 11: $437-468$.

Ingram, J. 1984. A new species of Argythamnia (Euphorbiaceae) from Mexico. Phytologia 55: 232-234.

Jablonski, E. 1915. Euphorbiaceae-Bridelieae. In: Engler, A. (ed.). Das Pflanzenreich IV. 147. (Heft 65): 1-98.

Jiménez, J. 1990. Una nueva sección y una especie nueva de Manihot (Euphorbiaceae) de México. An. Inst. Biol. Univ. Nac. Aut. Méx., Ser. Bot. 60: 49-53.

Jiménez, J. y M. J. Martínez. 2001. Una especie nueva del género Astrocasia (Euphorbiaceae) del estado de Guerrero. Acta Bot. Mex. 55: 1-5.

Jiménez, J., M. J. Martínez y R. Cruz. 2000. El género Plukenetia (Euphorbiaceae) en México. An. Inst. Biol. Univ. Nac. Aut. Méx., Ser. Bot. 71: 11-18.

Johnston, M. C. 1975. Studies of the Euphorbia species of the Chihuahuan Desert region and adjacent areas. Wrightia 5: 120-143.

Lomelí, J. A. y E. Sahagún. 1993. Pedilanthus diazlunanus (Euphorbiaceae), especie nueva del sur de Jalisco, México. Acta Bot. Mex. 25: 15-20.

Lundell, C. L. 1945. The genus Garcia Vahl, a potential source of superior hard quick-drying oil. Wrightia 1: $1-12$.

Martínez, M. J. 1996a. Contribución al conocimiento del género Croton (Euphorbiaceae), en el estado de Guerrero, México. Contr. Herb. Fac. Cien., Univ. Nac. Aut. Méx. 2. 109 pp.

Martínez, M. J. 1996b. El género Croton (Euphorbiaceae) en Mesoamérica. Tesis de maestría. Universidad Nacional Autónoma de México. México, D.F. 164 pp.

Martínez, M. J., J. Jiménez Ramírez. y R. Cruz Durán. 2000. El género Mabea (Euphorbiaceae) en México. An. Inst. Biol. Univ. Nac. Aut. Méx., Ser. Bot. 71: 87-95.

Mayfield, M. H. 1997. A systematic treatment of Euphorbia subgenus Poinsettia (Euphorbiaceae). Tesis de doctorado. University of Texas. Austin. $230 \mathrm{pp}$.

McVaugh, R. 1961. Euphorbiaceae novae Novo-Galicianae. Brittonia 13: 145-205.

McVaugh, R. 1993. Euphorbiae Novo-Galicianae revisae. Contr. Univ. Michigan Herb. 19: 207-239.

McVaugh, R. 1995. Euphorbiacearum sertum Novo-Galicianarum revisarum. Contr. Univ. Michigan Herb. 20: $173-215$ 
Müller (Argoviensis), J. 1866. Euphorbiaceae. In: de Candolle, Prodr. 15(2): 189-1261, 1269-1286.

Oudejans, R. C. H. M. 1990. World catalogue of species names published in the tribe Euphorbieae with their geographical distribution. Publicado por el autor, Utrecht. $444 \mathrm{pp}$.

Oudejans, R. C. H. M. 1993. World catalogue of species names published in the tribe Euphorbieae with their geographical distribution: cumulative supplement 1. Publicado por el autor, Utrecht. $78 \mathrm{pp}$.

Pax, F. 1910a. Euphorbiaceae-Jatropheae. In: Engler, A. (ed.). Das Pflanzenreich IV. 147. (Heft 42): 1-148.

Pax, F. 1910b. Euphorbiaceae-Adrianeae. In: Engler, A. (ed.). Das Pflanzenreich IV. 147. II. (Heft 44): 1-11

Pax, F. y K. Hoffmann. 1911. Euphorbiaceae-Cluytieae. In: Engler, A. (ed.). Das Pflanzenreich IV. 147. III. (Heft 47): 1-124.

Pax, F. y K. Hoffmann. 1912a. Euphorbiaceae-Gelonieae. In: Engler, A. (ed.). Das Pflanzenreich IV. 147. IV. (Heft 52): 1-41.

Pax, F. y K. Hoffmann. 1912b. Euphorbiaceae-Hippomaneae. In: Engler, A. (ed.). Das Pflanzenreich IV. 147. V. (Heft 52): 1-319.

Pax, F. y K. Hoffmann. 1912c. Euphorbiaceae-Acalypheae-Chrozophorinae. In: Engler, A. (ed.). Das Pflanzenreich IV. 147. VI. (Heft 57): 1-142.

Pax, F. y K. Hoffmann. 1914. Euphorbiaceae-Acalypheae-Mercurialinae. In: Engler, A. (ed.). Das Pflanzenreich IV. 147. VII. (Heft 63): 1-473.

Pax, F. y K. Hoffmann. 1919a. Euphorbiaceae-Acalypheae-Plukenetiinae-Epiprininae-Ricininae. In: Engler, A. (ed.). Das Pflanzenreich IV. 147. IX-XI. (Heft 68): 1-134.

Pax, F. y K. Hoffmann. 1919b. Euphorbiaceae-Dalechampieae. In: Engler, A. (ed.). Das Pflanzenreich IV. 147. XII. (Heft 68): 1-59.

Pax, F. y K. Hoffmann. 1919c. Euphorbiaceae-Pereae. In: Engler, A. (ed.). Das Pflanzenreich IV. 147. XIII. (Heft 68): 1-14.

Pax, F. y K. Hoffmann. 1919d. Euphorbiaceae-Additamentum VI. In: Engler, A. (ed.). Das Pflanzenreich IV. 147. XIV. (Heft 68): 1-81.

Pax, F. y K. Hoffmann. 1922. Euphorbiaceae-Phyllantheae. In: Engler, A. (ed.). Das Pflanzenreich IV. 147. XV. (Heft 81): 1-349.

Pax, F. y K. Hoffmann. 1924. Euphorbiaceae-Acalypheae-Acalyphinae-Additamentum VII-PhyllanthoideaeCrotonoideae. In: Engler, A. (ed.). Das Pflanzenreich IV. 147. XVI-XVII. (Heft 85): 1-231.

Radcliffe-Smith, A. 2001. Genera Euphorbiacearum. Royal Botanical Gardens. Kew, Surrey. 455 pp.

Radcliffe-Smith, A. y J. A. Ratter. 1996. A new Piranhea from Brazil, and the subsumption of the genus Celaenodendron (Euphorbiaceae: Oldfieldioideae). Kew Bull. 51: 543-548.

Ramírez R., M. A. 1997. Revisión taxonómica de Euphorbia subgénero Agaloma sección Alectoroctonum (Euphorbiaceae) en México. Tesis de maestría. Univeridad Nacional Autónoma de México. México, D.F. 135 pp.

Rogers, D. J. 1951. A revision of Stillingia in the New World. Ann. Missouri Bot. Gard. 38: 207-259.

Rogers, D. J. y S. G. Appan. 1973. Manihot and Manihotoides (Euphorbiaceae). Fl. Neotrop. Monogr. 13: $1-272$.

Rzedowski, J. 1978. Vegetación de México. Editorial Limusa. México, D.F. 432 pp.

Rzedowski, J. 1991. Diversidad y orígenes de la flora fanerogámica de México. Acta Bot. Mex. 14: 321.

Soltis, D. E., P. S. Soltis, M. W. Chase, M. E. Mort, D. C. Albach, M. Zanis, V. Savolainen, W. J. Hahn, S. B. Hoot, M. F. Fay, M. Axtell, S. M. Swensen, K. C. Nixon y J. S. Farris. 2000. Angiosperm phylogeny inferred from a combined data set of $18 \mathrm{~S} \mathrm{rDNA}, \mathrm{rbcL}$, and atpB sequences. Bot. J. Linn. Soc. 133: 381-461.

Standley, P. C. y J. A. Steyermark. 1949. Euphorbiaceae. In: Flora of Gualemala. Fieldiana Bot. 24(6): $25-170$ 
Steinmann, V. W. y R. S. Felger. 1997. The Euphorbiaceae of Sonora, Mexico. Aliso 16: 1-71.

Tokuoka, T. y H. Tobe. 1999. Embryology of tribe Drypeteae, an enigmatic taxon of Euphorbiaceae. Pl. Syst. Evol. 215: 189-208.

Urtecho, R. J. 1996. A taxonomic study of the Mexican species of Tragia (Euphorbiaceae). Tesis de doctorado. University of California. Davis. $358 \mathrm{pp}$.

Webster, G. L. 1965. A revision of the genus Meineckia (Euphorbiaceae). Acta Bot. Neerl. 14: 323365.

Webster, G. L. 1967. The genera of Euphorbiaceae in the southeastern United States. J. Arnold Arbor. 48: $303-430$.

Webster, G. L. 1975. Conspectus of a new classification of the Euphorbiaceae. Taxon 24: 593-601.

Webster, G. L. 1979. A revision of Margaritaria (Euphorbiaceae). J. Arnold Arbor. 60: 403-444.

Webster, G. L. 1992. Revision of Astrocasia (Euphorbiaceae). Syst. Bot. 17: 311-323.

Webster, G. L. 1994. Synopsis of the genera and suprageneric taxa of Euphorbiaceae. Ann. Missouri Bot. Gard. 81: 33-144.

Webster, G. L. 2001. Synopsis of Croton and Phyllanthus (Euphorbiaceae) in western tropical Mexico. Contr. Univ. Michigan Herb. 23: 353-388.

Webster, G. L. y M. J. Huft. 1988. Revised synopsis of Panamanian Euphorbiaceae. Ann. Missouri Bot. Gard. 75: 1087-1144.

Webster, G. L. y K. I. Miller. 1963. The genus Reverchonia (Euphorbiaceae). Rhodora 65: 193-207.

Wheeler, L. C. 1941. Euphorbia subgenus Chamaesyce in Canada and the United States exclusive of southern Florida. Rhodora 43: 97-154, 168-205, 223-286; plates 654-668.

Wheeler, L. C. 1960. Euphorbiaceae. In: Kearney, T. H. y R. H. Peebles. Arizona flora with supplement. University of California Press. Berkeley y Los Angeles. pp. 501-520; 1058-1059. 


\section{APÉNDICE 1}

Lista preliminar de los taxa de la familia Euphorbiaceae en México, ordenados de acuerdo con las subfamilias y tribus reconocidas por Webster (1994).

I. Subfamilia Phyllanthoideae

Tribu Wielandieae

(1) Savia Willd. (1 sp.)

1. S. sessiliflora (Sw.) Willd.; México a Sudamérica y en las Antillas.

Tribu Phyllantheae

(2) Astrocasia B. L. Rob. \& Millsp. (4 spp.)

2. A. diegoae J. Jiménez Ram. \& M. Martínez Gordillo; endémica de México.

3. A. neurocarpa (Müll. Arg.) I. M. Johnst. ex Standl.; endémica de México.

4. A. peltata Standl.; endémica de México.

5. A. tremula (Griseb.) G. L. Webster; México a Sudamérica y en las Antillas.

(3) Meineckia Baill. (2 spp.)

6. M. bartlettii (Standl.) G. L. Webster; Megaméxico 2.

7. M. neogranatensis (Müll. Arg.) G. L. Webster; México a Sudamérica.

(4) Andrachne L. (2 spp.)

8. A. arida (Warnock \& M. C. Johnst.) G. L. Webster; Megaméxico 1.

9. A. microphylla (Lam.) Baill. (A. ciliatoglandulosus (Millsp.) Croizat); México y de manera disyunta en Sudamérica (Perú).

(5) Margaritaria L. f. (1 sp.)

10. M. nobilis L. f. (Phyllanthus nobilis (L. f.) Müll. Arg.); México a Sudamérica y en las Antillas.

(6) Phyllanthus L. (41 spp.)

11. P. abnormis Baill. var. abnormis; Estados Unidos a México.

12. P. acidus (L.) Skeels; introducida de Brazil.

13. P. acuminatus Vahl ( $P$. sessei Briq.); México a Sudamérica y en las Antillas.

14. P. adenodiscus Müll. Arg.; endémica de México.

15. P. amarus Schumach. \& Thonn.; Estados Unidos a Sudamérica y en las Antillas, introducida en el Viejo Mundo.

16. P. barbarae M. C. Johnst.; endémica de México.

17. P. botryanthus Müll. Arg.; México a Sudamérica y en las Antillas.

18. P. brandegei Millsp.; endémica de México.

19. P. caribaeus Urb.; México a Sudamérica y en las Antillas.

20. P. caroliniensis Walter var. caroliniensis; Estados Unidos a Sudamérica.

21. P. chiapensis Sprague; endémica de México.

22. P. coalcomanensis Croizat; endémica de México.

23. P. compressus H.B.K.; México a Sudamérica.

24. P. elsiae Urb.; México a Sudamérica y en las Antillas.

25. P. ericoides Torr.; Megaméxico 1.

26. P. evanescens Brandegee; Megaméxico 3.

27. P. fluitans Benth. ex Müll. Arg.; México y de manera disyunta en Sudamérica.

28. P. fraguensis M. C. Johnst.; endémica de México.

29. P. galeottianus Baill.; endémica de México.

30. P. grandifolius L.; endémica de México.

31. P. gypsicola McVaugh; endémica de México.

32. P. harrimanii G. L. Webster; endémica de México. 
Apéndice 1. Continuación.

33. P. hexadactylus McVaugh; endémica de México.

34. P. liebmannianus Müll. Arg. var. liebmannianus; Megaméxico 2.

35. P. mcvaughii G. L. Webster; Megaméxico 2.

36. P. mickelii McVaugh; endémica de México.

37. P. micrandrus Müll. Arg.; México a Sudamérica.

38. P. mocinianus Baill.; Megaméxico 2.

39. $P$. neoleonensis Croizat; endémica de México.

40. P. niruri L.; Estados Unidos a Sudamérica y en las Antillas.

41. P. oaxacanus Brandegee; endémica de México.

42a. P. peninsularis Brandegee ssp. novogalicianus G. L. Webster; endémica de México.

42b. P. peninsularis Brandegee ssp. peninsularis; endémica de México.

43. P. petaloideus P. G. Wilson; endémica de México.

44. $P$. polygonoides Nutt. ex Spreng.; Estados Unidos a México.

45. P. purpusii Brandegee; endémica de México.

46. P. standleyi McVaugh; endémica de México.

47. P. stipulatus (Raf.) G. L. Webster; México a Sudamérica y en las Antillas.

48. P. subcuneatus Greenm.; endémica de México.

49. P. tenellus Roxb.; introducida de África.

50. P. tequilensis B. L. Rob. \& Greenm. ( $P$. micromalus McVaugh); endémica de México.

51. P. tuerckheimii G. L. Webster; Megaméxico 2.

(7) Reverchonia A. Gray. (1 sp.)

52. R. arenaria A. Gray; Estados Unidos a México.

Tribu Antidesmeae

(8) Hyeronima Allemão (3 spp.)

53. H. alchorneoides Allemão; México a Sudamérica.

54. H. fendleri Briq.; México a Sudamérica.

55. H. oblonga (Tul.) Müll. Arg.; México a Sudamérica.

II. Subfamilia Oldfieldioideae

Tribu Podocalyceae

(9) Tetracoccus Engelm. ex Parry (4 spp.)

56. T. capensis (I. M. Johnst.) Croizat; endémica de México.

57. T. dioicus Parry; Megaméxico 1.

58. T. fasciculatus (S. Watson) Croizat; endémica de México.

59. T. hallii Brandegee (T. fasciculatus var. hallii (Brandegee) Dressler); Megaméxico 1.

Tribu Picrodendreae

(10) Piranhea Baill. (=Celaenodendron Standl.) (1 sp.)

60. P. mexicana (Standl.) Radcl.-Sm. (C. mexicanum Standl.); endémica de México.

III. Subfamilia Acalyphoideae

Tribu Pereae

(11) Pera Mutis (1 sp.)

61. P. barbellata Standl.; Megaméxico 2. 
Apéndice 1. Continuación.

Tribu Chrozophoreae

(12) Caperonia St.-Hil. (3 spp.)

62. C. castaneifolia (L.) St.-Hil.; México a Sudamérica y en las Antillas.

63. C. chiltepecensis Croizat; endémica de México.

64. C. palustris (L.) St. Hil.; Estados Unidos a Sudamérica y en las Antillas.

(13) Ditaxis Vahl ex A. Juss. (15 spp.)

65. D. adenophora (A. Gray) Pax \& K. Hoffm.; Megaméxico 1.

66. D. arlynniana (J. W. Ingram) Radcl.-Sm. \& Govaerts; endémica de México.

67a. D. brandegeei (Millsp.) Rose \& Standl. var. brandegeei; endémica de México.

67b. D. brandegeei (Millsp.) Rose \& Standl. var. intonsa I. M. Johnst.; Megaméxico 1.

68. D. clariana (Jeps.) G. L. Webster; Megaméxico 1.

69. D. depressa (Greenm.) Pax \& K. Hoffm.; endémica de México.

70. D. dressleriana (J. W. Ingram) Radcl.-Sm. \& Govaerts; endémica de México.

71. D. guatemalensis (Müll. Arg.) Pax \& K. Hoffm. (D. guatemalensis var. barrancana (McVaugh) Radcl.-Sm. \& Govaerts); México a Centroamérica.

72. D. heterantha Zucc.; endémica de México.

73. D. humilis (Engelm. \& A. Gray) Pax; Estados Unidos a México.

74. D. lanceolata (Benth.) Pax \& K. Hoffm.; Megaméxico 1.

75. D. manzanilloana (Rose) Pax \& K. Hoffm.; endémica de México.

76. D. micrandra (Croizat) Radcl.-Sm. \& Govaerts; endémica de México.

77. D. neomexicana (Müll. Arg.) A. Heller; Estados Unidos a México.

78. D. pringlei (Greenm.) Pax \& K. Hoffm.; endémica de México.

79. D. serrata (Torr.) A. Heller; Megaméxico 1.

(14) Argythamnia P. Brown (7 spp.)

80. A. coatepensis (Brandegee) Croizat; endémica de México.

81. A. lottiae J. W. Ingram; endémica de México.

82. A. lundellii J. W. Ingram; endémica de México.

83. A. moorei J. W. Ingram; endémica de México.

84. A. sitiens (Brandegee) J. W. Ingram; endémica de México.

85. A. tinctoria Millsp.; endémica de México.

86. A. wheeleri J. W. Ingram; endémica de México.

(15) Chiropetalum Adr. Jussieu (2 spp.)

87. C. astroplethes (J. W. Ingram) Radcl.-Sm. \& Govaerts; endémica de México.

88. C. schiedeanum (Müll. Arg.) Pax ex Pax \& K. Hoffm.; endémica de México.

Tribu Bernardieae

(16) Bernardia Houst. ex Mill. (25 spp.)

89. B. albida Lundell; endémica de México.

90. B. aspera Pax \& K. Hoffm.; endémica de México.

91. B. chiapensis Lundell; endémica de México.

92. B. dodecandra (Sessé ex Cav.) McVaugh (B. interrupta Schltdl.); Megaméxico 2.

93. B. fonsecae A. Cervantes \& J. Jiménez Ram.; endémica de México.

94. B. gentryana Croizat; endémica de México.

95. B. heteropilosa McVaugh; endémica de México.

96. B. incana J. K. Morton; Megaméxico 1.

97. B. kochii McVaugh; endémica de México.

98. B. lagunensis (M. E. Jones) L. C. Wheeler; endémica de México.

99. B. mexicana (Hook. \& Arn.) Müll. Arg.; endémica de México. 
Apéndice 1. Continuación.

100. B. mollis Lundell; Megaméxico 2.

101. B. myricifolia (Scheele) S. Watson; Megaméxico 1.

102. B. oblanceolata Lundell; Megaméxico 2.

103. B. obovata I. M. Johnst.; Megaméxico 1.

104. B. ovalifolia Lundell; endémica de México.

105. B. santanae McVaugh; endémica de México.

106. B. sidoides (Klotzsch) Müll.Arg.; introducida de Sudamérica.

107. B. spongiosa McVaugh; endémica de México.

108. B. viridis Millsp.; endémica de México.

109. B. wilburii McVaugh; endémica de México.

110. B. yucatanensis Lundell; Megaméxico 2.

111. B. sp. nov. 1; endémica de México.

112. B. sp. nov. 2; endémica de México.

113. B. sp. nov. 3; endémica de México.

Tribu Adelieae

(17) Adelia L. (6 spp.)

114. A. barbinervis Cham. \& Schltdl.; Megaméxico 2.

115. A. brandegeei V. W. Steinm. (=A. virgata Brandegee); endémica de México.

116. A. cinerea (Wiggins \& Rollins) A. Cervantes, V. W. Steinm. \& H. Flores; endémica de México

117. A. oaxacana (Müll. Arg.) Hemsl.; endémica de México.

118. A. obovata Wiggins \& Rollins; endémica de México.

119. A. vaseyi (Coult.) Pax \& K. Hoffm.; Megaméxico 1.

(18) Enriquebeltrania Rzedowski (1 sp.)

120. E. crenatifolia (Miranda) Rzedowski; endémica de México.

Tribu Alchorneae

(19) Alchornea Sw. (3 spp.)

121. A. chiapasana Miranda; Megaméxico 2.

122. A. latifolia Sw.; México a Sudamérica y en las Antillas.

123. A. similis Müll. Arg.; endémica de México.

Tribu Acalypheae

(20) Ricinus L. (1 sp.)

124. R. communis L.; introducida de África.

(21) Cleidion Blume (1 sp.)

125. C. castaneifolium Müll. Arg.; México a Sudamérica.

(22) Acalypha L. (108 spp.)

126. A. adenostachya Müll. Arg.; endémica de México.

127. A. aliena Brandegee (A. simplicissima Millsp.); Megaméxico 2.

128. A. alopecuroidea Jacq.; México a las Antillas y Sudamérica.

129. A. anadenia Standl.; endémica de México.

130. A. aristata H.B.K. (A. arvensis Poepp. \& Endl.); México a Sudamérica.

131. A. botteriana Müll. Arg.; endémica de México.

132. A. brevicaulis Müll. Arg.; endémica de México.

133. A. burquezii V. W. Steinm. \& Felger; endémica de México.

134. A. californica Benth. (A. pringlei S. Watson); Megaméxico 1. 
Apéndice 1. Continuación.

135. A. chiapensis Brandegee; endémica de México.

136. A. cincta Müll. Arg. (A. gentryi Standl.); endémica de México.

137. A. cinerea Pax \& K. Hoffm.; endémica de México.

138. A. coleispica Pax \& K. Hoffm.; endémica de México.

139. A. comonduana Millsp.; endémica de México.

140. A. confertiflora Pax \& K. Hoffm.; endémica de México.

141. A. conspicua Müll. Arg.; endémica de México.

142. A. costaricensis (Kuntze) Knobl.; México a Centroamérica.

143. A. delgadoana McVaugh; endémica de México.

144. A. depauperata Müll. Arg.; endémica de México.

145. A. dioica S. Watson ( $A$. dissitiflora $\mathrm{S}$. Watson); endémica de México.

146. A. diversifolia Jacq. (A. tabascensis Lundell); México a Sudamérica.

147. A. euphrasiostachys Bartlett; Megaméxico 2.

148. A. ferdinandii K. Hoffm.; Megaméxico 2.

149. A. filipes (S. Watson) McVaugh (A. coryloides Rose); endémica de México.

150. A. flagellata Millsp.; endémica de México.

151. A. fournieri Müll. Arg.; endémica de México.

152. A. fredericii Müll. Arg.; endémica de México.

153. A. gaumeri Pax \& K. Hoffm.; endémica de México.

154. A. gigantesca McVaugh; endémica de México.

155. A. glandulosa Cav.; México y de manera disyunta en Sudamérica (Colombia).

156. A. grisea Pax \& K. Hoffm.; endémica de México.

157. A. gummifera Lundell; Megaméxico 2.

158. A. haploclada Pax \& K. Hoffm.; endémica de México.

159. A. hypogaea S. Watson; endémica de México.

160. A. indica L.; introducida del Viejo Mundo.

161. A. infesta Poepp. \& Endl. (A. jerzedowskii Calderón); introducida de Sudamérica.

162. A. karwinskii Müll. Arg.; endémica de México.

163. A. katharinae Pax; endémica de México.

164. A. lagascana Müll. Arg.; endémica de México.

165. A. lagopus McVaugh; endémica de México.

166a. A. langiana Müll. Arg. var. langiana; Megaméxico 2.

166b. A. langiana Müll. Arg. var. vigens McVaugh; endémica de México.

167. A. lancetillae Standl.; Megaméxico 2.

168. A. laxiflora Müll. Arg.; México y las Antillas.

169. A. leptopoda Müll. Arg. (A. unibracteata Müll. Arg.); México a Centroamérica.

170. A. liebmanniana Müll. Arg.; endémica de México.

171. A. lignosa Brandegee; endémica de México.

172. A. lindeniana Müll. Arg.; endémica de México.

173. A. longispicata Müll. Arg.; endémica de México.

174. A. longistipularis Müll. Arg.; endémica de México.

175. A. lovelandii (McVaugh) McVaugh; endémica de México.

176. A. macrostachya Jacq.; México a Sudamérica.

177. A. macrostachyoides Müll. Arg.; endémica de México.

178. A. melochiifolia Müll. Arg.; endémica de México.

179. A. mexicana Müll. Arg. (A. indica L. var. mexicana (Müll. Arg.) Pax \& K. Hoffm.); México a Centroamérica.

180. A. microcephala Müll. Arg.; endémica de México. 
Apéndice 1. Continuación.

181a. A. microphylla Klotzsch var. interior McVaugh; endémica de México.

181b. A. microphylla Klotzsch var. microphylla; Megaméxico 2.

182. A. mollis H.B.K.; endémica de México.

183. A. monostachya Cav. (A. amenioides H.B.K., A. hederacea Torr.); Megaméxico 1.

184. A. multiflora (Standl.) Radcl.-Sm.; endémica de México.

185. A. multispicata $\mathrm{S}$. Watson; endémica de México.

186a. A. neomexicana Müll. Arg. var. jaliscana McVaugh (A. salvadorensis Standl.); Megaméxico 2.

186b. A. neomexicana Müll. Arg. var. neomexicana; Megaméxico 1.

187. A. nubicola McVaugh; endémica de México.

188. A. ocymoides H.B.K.; endémica de México.

189. A. oligantha Müll. Arg.; endémica de México.

190. A. oligodonta Müll. Arg.; endémica de México.

191. A. oreopola Greenm.; endémica de México.

192. A. ostryifolia Ridd.; Estados Unidos a Centroamérica y en las Antillas.

193. A. papillosa Rose; endémica de México.

194. A. phleoides Cav. Megaméxico 3.

195. A. pilosa Cav.; Megaméxico 2.

196. A. pippenii McVaugh; endémica de México.

197. A. poiretii Spreng.; México a Sudamérica y en las Antillas.

198. A. polystachya Jacq.; México a Sudamérica.

199. A. pseudalopecuroides Pax \& K. Hoffm.; Megaméxico 2.

200. A. pseudovagans Pax \& K. Hoffm.; endémica de México.

201. A. purpurascens H.B.K.; endémica de México.

202. A. purpusii Brandegee; endémica de México.

203. A. radians Torr.; Megaméxico 1.

204. A. rafaelensis Standl.; endémica de México.

205. A. rhombifolia Schltdl.; endémica de México.

206. A. rubroserrata Pax \& K. Hoffm.; endémica de México.

207. A. sabulicola Brandegee; endémica de México.

208. A. saxicola Wiggins; endémica de México.

209. A. schiedeana Schltdl.; México a Sudamérica.

210. A. schlechtendaliana Müll. Arg.; endémica de México.

211. A. schlumbergeri Müll. Arg.; endémica de México.

212. A. seleriana Greenm.; endémica de México.

213. A. setosa A. Rich.; México a Sudamérica.

214. A. skutchii I. M. Johnst.; Megaméxico 2.

215. A. subterranea P. G. Wilson; endémica de México.

216. A. subtomentosa Lag.; endémica de México.

217. A. subviscida S. Watson; Megaméxico 2.

218. A. synoica Pax \& K. Hoffm.; Megaméxico 2.

219. A. tacanensis Lundell; Megaméxico 2.

220. A. tamaulipasensis Lundell; endémica de México.

221. A. tenuicauda Pax \& K. Hoffm.; Megaméxico 2.

222. A. trachyloba Müll. Arg. (A. glandulifera B. L. Rob. \& Greenm.); endémica de México.

223. A. tricholoba Müll. Arg.; Megaméxico 2

224. A. trilaciniata P. G. Wilson; endémica de México.

225. A. triloba Müll. Arg.; Megaméxico 2. 
Apéndice 1. Continuación.

226. A. umbrosa Brandegee; endémica de México.

227. A. vagans Cav.; endémica de México.

228. A. vallartae McVaugh; endémica de México.

229. A. veronicoides Pax \& K. Hoffm.; endémica de México.

230. A. villosa Jacq.; México a Sudamérica.

231. A. sp. nov. 1; endémica de México.

232. A. sp. nov. 2; endémica de México.

233. A. sp. nov. 3; endémica de México.

Tribu Plukenetieae

(23) Plukenetia L. (3 spp.)

234. P. carabiasiae J. Jiménez Ram.; endémica de México.

235. P. penninervia Müll. Arg.; México a Sudamérica.

236. P. stipellata L. J. Gillespie; México a Centroamérica.

(24) Tragia Plumier ex L. (20 spp.)

237. T. affinis B. L. Rob. \& Greenm.; endémica de México.

238. T. amblyodonta (Müll. Arg.) Pax \& K. Hoffm.; Megaméxico 1.

239. T. bailloniana Müll. Arg.; México a Centoamérica.

240. T. brevispica Engelm. \& A. Gray; Megaméxico 1.

241. T. glanduligera Pax \& K. Hoffm. (T. jonesii Radcl.-Sm. \& Govaerts); Megaméxico 1.

242. T. laciniata (Torr.) Müll. Arg.; Megaméxico 1.

243. T. mexicana Müll. Arg.; Megaméxico 2.

244a. T. nepetifolia Cav. var. dissecta Müll. Arg.; Megaméxico 1.

244b. T. nepetifolia Cav. var. nepetifolia; Megaméxico 1.

244c. T. nepetifolia Cav. var. setosa S. Watson; endémica de México.

245. T. pacifica McVaugh; endémica de México.

246. T. potosina Lundell; endémica de México.

247. T. ramosa Torr.; Estados Unidos a México.

248. T. volubilis L.; México a Sudamérica y en las Antillas; introducida en África.

249. T. yucatanensis Millsp.; endémica de México.

250. T. sp. nov. 1; endémica de México.

251. T. sp. nov. 2; endémica de México.

252. T. sp. nov. 3; endémica de México.

253. T. sp. nov. 4; endémica de México.

254. T. sp. nov. 5; endémica de México.

255. T. sp. nov. 6; endémica de México.

256. T. sp. nov. 7; endémica de México.

(25) Dalechampia Plumier ex L. (6 spp.)

257. D. heteromorpha Pax \& K. Hoffm.; México a Centroamérica.

258. D. magnistipulata G. L. Webster \& Armbr.; endémica de México.

259. D. scandens L. var. scandens; México a Sudamérica y en las Antillas.

260. D. schottii Greenm.; endémica de México.

261. D. spathulata (Scheidw.) Baill.; México a Sudamérica.

262. D. tiliifolia Lam.; México a Sudamérica.

Tribu Omphaleae

(26) Omphalea L. (1 sp.)

263. O. oleifera HemsI. (O. cardiophylla Hemsl.); Megaméxico 2. 
Apéndice 1. Continuación.

IV. Subfamilia Crotonoideae

Tribu Manihoteae

(27) Manihot Mill. (Manihotoides D. J. Rogers \& Appan) (20 spp.)

264. M. aesculifolia (H.B.K.) Pohl; México a Centroamérica.

265. M. angustiloba (Torr.) Müll. Arg.; Megaméxico 1.

266. M. auriculata McVaugh; endémica de México.

267. M. caudata Greenm.; endémica de México.

268. M. chlorosticta Standl. \& Goldman; endémica de México.

269. M. crassisepala Pax \& K. Hoffm.; endémica de México.

270. M. davisae Croizat; Megaméxico 1.

271. M. foetida (H.B.K.) Pohl; endémica de México.

272. M. michaelis McVaugh; endémica de México.

273. M. oaxacana D. J. Rogers \& Appan; endémica de México.

274. M. obovata J. Jiménez Ram.; endémica de México.

275. M. pauciflora Brandegee (Manihotoides pauciflora (Brandegee) D. J. Rogers \& Appan); endémica de México.

276. M. pringlei S. Watson; endémica de México.

277a. M. rhomboidea Müll. Arg. ssp. microcarpa (Müll. Arg.) D. J. Rogers \& Appan; Megaméxico 2

277b. M. rhomboidea Müll. Arg. ssp. rhomboidea endémica de México.

278. M. rubricaulis I. M. Johnst. ( $M$. isoloba Standl.); endémica de México.

279. M. subspicata D. J. Rogers \& Appan; endémica de México.

280. M. tomatophylla Standl.; endémica de México.

281. M. walkerae Croizat; Megaméxico 1.

282. M. websteri D. J. Rogers \& Appan; endémica de México.

283. M. sp. nov.; endémica de México.

(28) Cnidoscolus Pohl (24 spp.)

284a. C. aconitifolius (Mill.) I. M. Johnst. var. aconitifolius (C. chaya Lundell, C. chayamansa McVaugh); Megaméxico 2.

284b. C. aconitifolius (Mill.) I. M. Johnst. var. polyanthus (Pax \& K. Hoffm.) Breckon; endémica de México.

285. C. albidus Lundell; endémica de México.

286. C. angustidens Torr.; Megaméxico 1.

287. C. autlanensis Breckon; endémica de México.

288. C. calyculatus (Pax \& K. Hoffm.) I. M. Johnst.; endémica de México.

289. C. egregius Breckon; endémica de México.

290. C. elasticus Lundell; endémica de México.

291. C. herbaceus (L.) I. M. Johnst.; endémica de México.

292. C. jurgensenii (Briq.) Lundell; Megaméxico 2.

293. C. liebmannii (Müll. Arg.) Lundell; endémica de México.

294. C. maculatus (Brandegee) Pax \& K. Hoffm.; endémica de México.

295. C. multilobus (Pax) I. M. Johnst.; endémica de México.

296. C. orbiculatus Lundell; endémica de México.

297. C. palmeri (S. Watson) Rose; endémica de México.

298a. C. rostratus Lundell ssp. glabratus Breckon; endémica de México.

298b. C. rostratus Lundell ssp. hintonii Breckon; endémica de México.

298c. C. rostratus Lundell ssp. rostratus; endémica de México. 
Apéndice 1. Continuación.

299. C. rotundifolius (Müll. Arg.) McVaugh; endémica de México.

300. C. shrevei I. M. Johnst.; endémica de México.

301. C. souzae McVaugh; endémica de México.

302. C. spinosus Lundell; endémica de México.

303. C. tehuacanensis Breckon; endémica de México.

304. C. tepiquensis (Costantin \& Gallaud) Lundell; endémica de México.

305. C. urens (L.) Arthur; Estados Unidos a Sudamérica y también en las Antillas.

306. C. sp. nov. 1; endémica de México.

307. C. sp. nov. 2; Megaméxico 2.

Tribu Adenoclineae

(29) Tetrorchidium Poepp. (2 spp.)

308. T. brevifolium Standl. \& Steyerm.; Megaméxico 2.

309. T. rotundatum Standl.; México a Centroamérica.

Tribu Jatropheae

(30) Jatropha L. (48 spp.)

310. J. alamanii Müll. Arg.; endémica de México.

311. J. andrieuxii Müll. Arg.; endémica de México.

312. J. bartlettii Wilbur; endémica de México.

313. J. bullockii E. J. Lott; endémica de México.

314. J. cardiophylla (Torr.) Müll. Arg.; Megaméxico 1.

315. J. cathartica Terán \& Berland.; Megaméxico 1.

316. J. chamelensis Pérez-Jim.; endémica de México.

317. J. ciliata Sessé ex Cerv.; endémica de México.

318. J. cinerea (Ort.) Müll. Arg. (Jatropha canescens (Benth.) Müll. Arg.); Megaméxico 1.

319. J. contrerasii J. Jiménez Ram. \& M. Martínez Gordillo; endémica de México.

320. J. conzattii J. Jiménez Ram.; endémica de México.

321. J. cordata (Ort.) Müll. Arg.; endémica de México.

322. J. cuneata Wiggins \& Rollins; Megaméxico 1.

323. J. curcas L. (Jatropha mcvaughii Dehgan \& G. L. Webster); México a Sudamérica y en las Antillas.

324. J. dehganii J. Jiménez Ram.; endémica de México.

325. J. dioica Cerv.; Megaméxico 1.

326. J. elbae J. Jiménez Ram.; endémica de México.

327. J. fremontioides Standl.; endémica de México.

328. J. galvanii J. Jiménez Ram.; endémica de México.

329. J. gaumeri Greenm.; Megaméxico 2.

330. J. giffordiana Dehgan \& G. L. Webster; endémica de México.

331. J. gossypifolia L.; México a Sudamérica y en las Antillas.

332. J. hintonii Wilbur; endémica de México.

333. J. krusei J. Jiménez Ram. y M. Martínez Gordillo; endémica de México.

334a. J. macrorhiza Benth. var. macrorhiza; endémica de México.

334b. J. macrorhiza Benth. var. septemfida Engelm.; Megaméxico 1.

335. J. malacophylla Standl.; endémica de México.

336. J. moranii Dehgan \& G. L. Webster; endémica de México.

337. J. neopauciflora Pax; endémica de México.

338. J. oaxacana J. Jiménez Ram. \& R. Torres; endémica de México. 
Apéndice 1. Continuación.

339. J. octandra Cerv.; endémica de México.

340. J. ortegae Standl.; endémica de México.

341. J. peltata Cerv. (J. platyphylla Müll. Arg.); endémica de México.

342. J. pereziae J. Jiménez Ram.; endémica de México.

343. J. pseudocurcas Müll. Arg.; endémica de México.

344. J. purpurea Rose; endémica de México.

345. J. riojae Miranda; endémica de México.

346. J. rufescens Brandegee; endémica de México.

347. J. rzedowskii J. Jiménez Ram.; endémica de México.

348. J. standleyi Steyerm.; endémica de México.

349. J. stephanii J. Jiménez Ram. endémica de México.

350. J. sympetala S. F. Blake \& Standl.; endémica de México.

351. J. tehuantepecana J. Jiménez Ram. \& A. Campos; endémica de México.

352. J. tlalcozotitlanensis J. Jiménez Ram.; endémica de México.

353. J. vernicosa Brandegee; endémica de México.

354. J. websteri J. Jiménez Ram.; endémica de México.

355. J. sp. nov. 1; endémica de México.

356. J. sp. nov. 2; endémica de México.

357. J. sp. nov. 3; endémica de México.

Tribu Codiaeae

(31) Acidocroton Griseb. (Ophellantha Standl.) (3 spp.)

358. A. spinosus (Standl.) G. L. Webster (O. spinosa Standl.); Megaméxico 2.

359. A. steyermarkii (Standl.) G. L. Webster (O. steyermarkii Standl.); Megaméxico 2.

360. A. sp. nov.; endémica de México.

Tribu Crotoneae

(32) Croton L. (Julocroton Mart.; Eremocarpus Benth.) (124 spp.)

361. C. acapulcensis M. Martínez \& J. Jiménez Ram.; endémica de México.

362. C. adspersus Benth.; Megaméxico 2.

363. C. alamosanus Rose; endémica de México.

364. C. ameliae Lundell; endémica de México.

365. C. amphileucus Briq.; endémica de México.

366. C. arboreus Millsp.; endémica de México.

367. C. argenteus L. (Julocroton argenteus (L.) Didr.); Estados Unidos a Sudamérica.

368. C. axillaris Müll. Arg.; México a Centroamérica.

369. C. billbergianus Müll. Arg. ssp. pyramidalis (Donn. Sm.) G. L. Webster; México a Centroamérica.

370. C. caboensis Croizat; endémica de México.

371. C. californicus Müll. Arg.; Estados Unidos a México.

372. C. campechianus Standl.; endémica de México.

373. C. capitatus Michx.; Estados Unidos a México.

374. C. chamelensis E. J. Lott; endémica de México.

375. C. chiapensis Lundell; endémica de México.

376. C. chichenensis Lundell; endémica de México.

377. C. ciliatoglandulifer Ort.; Megaméxico 3.

378. C. conspurcatus Schltdl. (Julocroton conspurcatus (Schltdl.) Klotzsch); Megaméxico 2.

379. C. cortesianus H.B.K.; Megaméxico 3. 
Apéndice 1. Continuación.

380. C. culiacanensis Croizat; endémica de México.

381. C. cupulifer McVaugh; endémica de México.

382. C. decalobus Müll. Arg.; México a Centroamérica.

383. C. dioicus Cav.; Megaméxico 1.

384. C. disjunctus V. W. Steinm.; endémica de México.

385. C. draco Schltdl.; México a Centroamérica.

386. C. ehrenbergii Schltdl.; endémica de México.

387. C. fantzianus Seymour; Megaméxico 2.

388. C. flavens L.; México y en las Antillas.

389. C. flavescens Greenm.; endémica de México.

390. C. flaviglandulosus Lundell; endémica de México.

391. C. fragilis H.B.K.; México a Sudamérica.

392. C. francoanus Müll. Arg.; Megaméxico 2.

393. C. fruticulosus Torr.; Megaméxico 1.

394. C. gaumeri Millsp.; endémica de México.

395. C. glabellus L. (C. nitens Sw.); México a Sudamérica y en las Antillas.

396. C. glandulosepalus Millsp.; Megaméxico 2.

397. C. glandulosus L.; Estados Unidos a Sudamérica y en las Antillas.

398. C. gossypifolius Vahl; México a Sudamérica.

399. C. grewiifolius Müll. Arg.; endémica de México.

400. C. guatemalensis Lotsy (C. wilburii McVaugh); Megaméxico 2.

401. C. gynopetalus Croizat; endémica de México.

402. C. hirtus L'Hér.; México a Sudamérica y en las Antillas.

403. C. hoffmannii Müll. Arg.; México a Centroamérica.

404. C. huajuapanensis M. Martínez Gordillo \& Cruz-Durán; endémica de México.

405. C. humilis L.; Estados Unidos a México y en las Antillas.

406. C. iche Lundell; endémica de México.

407. C. incanus H.B.K.; endémica de México.

408. C. itzaeus Lundell; endémica de México.

409. C. juncundus Brandegee; endémica de México.

410. C. jutiapensis Croizat; México a Centroamérica.

411. C. lasiopetalus Croizat; Megaméxico 2.

412. C. leucophyllus Müll. Arg.; Megaméxico 1.

413. C. liebmannii Müll. Arg.; endémica de México.

414a. C. lindheimerianus Scheele var. lindheimerianus; Estados Unidos a México.

414b. C. lindheimerianus Scheele var. tharpii M. C. Johnst.; Megaméxico 1.

415. C. lobatus L.; Estados Unidos a Sudamérica y en las Antillas.

416. C. lundellii Standl.; Megaméxico 2.

417. C. macrodontus Müll. Arg.; endémica de México.

418. C. magdalenae Millsp.; endémica de México.

419. C. malvaviscifolius Millsp.; endémica de México.

420. C. martinianus V. W. Steinm.; endémica de México.

421. C. masonii I. M. Johnst.; endémica de México.

422. C. matudai Lundell; endémica de México.

423a. C. mazapensis Lundell var. mazapensis; endémica de México.

423b. C. mazapensis Lundell var. obtusifolius (Müll. Arg.) G. L. Webster (C. rzedowskii M. C. Johnst.); endémica de México.

423c. C. mazapensis Lundell var. pacificus G. L. Webster; endémica de México. 
Apéndice 1. Continuación.

424. C. mcvaughii G. L. Webster; endémica de México.

425. C. meissneri Müll. Arg.; endémica de México.

426. C. mexicanus Müll. Arg.; México a Centroamérica.

427. C. michaelii V. W. Steinm.; endémica de México.

428. C. millspaughii Standl.; endémica de México.

429. C. miradorensis Müll. Arg.; endémica de México.

430. C. monanthogynus Michx.; Estados Unidos a México.

431a. C. morifolius Willd. var. morifolius (C. sericeus Schltdl. \& Cham., C. deppeanus Steud.); México a Sudamérica.

431b. C. morifolius Willd. var. sphaerocarpus (H.B.K.) Müll. Arg. (C. sphaerocarpus H.B.K.); México a Sudamérica.

432. C. muelleri Coult.; endémica de México.

433. C. niveus Jacq. (C. septemnervius McVaugh); México a Sudamérica.

434. C. ortegae Standl.; endémica de México.

435. C. ortholobus Müll. Arg.; México a Centroamérica.

436. C. ovalifolius Vahl (C. escathos Croizat); Mexico a Sudamérica y en las Antillas.

437. C. palmeri S. Watson; endémica de México.

438. C. pedicellatus H.B.K. (C. tenuilobus S. Watson); México y de manera disyunta en Sudamérica.

439. C. pendens Lundell; Megaméxico 2

440. C. peraeruginosus Croizat; endémica de México.

441. C. pottsii (Klotzsch) Müll. Arg.; Megaméxico 1.

442. C. pseudoglabellus Lundell; endémica de México.

443. C. pseudoniveus Lundell; México a Centroamérica.

444. C. pulcher Müll. Arg.; endémica de México.

445. C. punctatus Jacq.; Estados Unidos a Centroamérica y en las Antillas.

446. C. pyriticus Croizat; México a Centroamérica.

447a. C. ramillatus Croizat ssp. magniglandulifer V. W. Steinm.; endémica de México.

447b. C. ramillatus Croizat ssp. ramillatus; endémica de México.

448. C. reflexifolius H.B.K.; Megaméxico 2.

449. C. repens Schltdl.; Megaméxico 2.

450. C. rhamnifolius H.B.K.; México a Sudamérica.

451. C. rivinifolius H.B.K.; México a Sudamérica.

452. C. roxanae Croizat; endémica de México.

453. C. sancti-lazarii Croizat; Megaméxico 1.

454. C. schiedeanus Schltdl.; México a Sudamérica.

455. C. setiger Hook. (Eremocarpus setiger (Hook.) Benth.); Estados Unidos a México.

456. C. shreveanus Croizat; endémica de México.

457. C. siltepecensis Lundell; endémica de México.

458. C. soliman Cham. \& Schltdl.; Megaméxico 1.

459. C. sonorae Torr. (C. pringlei S. Watson, C. attenuatus M. E. Jones); Megaméxico 1.

460. C. sousae M. Martínez Gordillo \& Cruz-Durán; endémica de México.

461. C. stenopetalus G. L. Webster; endémica de México.

462. C. stipulaceus H.B.K.; endémica de México.

463. C. suaveolens Torr.; Megaméxico 1.

464. C. suberosus H.B.K. (C. cladotrichus Müll. Arg., C. purpusii Brandegee); endémica de México.

465. C. subfragilis Müll. Arg.; endémica de México. 
Apéndice 1. Continuación.

466. C. subjuncundus Croizat; endémica de México.

467. C. sutup Lundell; endémica de México.

468. C. tabascensis Lundell; endémica de México.

469. C. texensis (Klotzsch) Müll. Arg.; Estados Unidos a México.

470. C. tremulifolius Croizat; endémica de México.

471. C. trinitatis Millsp.; Estados Unidos a Sudamérica.

472. C. varelae V. W. Steinm.; endémica de México.

473. C. verapazensis Donn. Sm.; Megaméxico 2.

474. C. virletianus Müll. Arg.; endémica de México.

475. C. watsonii Standl.; endémica de México.

476. C. websteri M. Martínez Gordillo \& J. Jiménez Ram.; endémica de México.

477. C. wigginsii L. C. Wheeler; Megaméxico 1.

478. C. xalapensis H.B.K.; México a Centroamérica.

479. C. yecorensis V. W. Steinm. \& Felger; endémica de México.

480. C. ynesae Croizat; endémica de México.

481. C. yucatanensis Lundell; México a Centroamérica.

482. C. zavaletae Conz. ex Rzed. et al.; endémica de México.

483. C. sp. nov. 1; endémica de México.

484. C. sp. nov. 2; endémica de México.

Tribu Aleuritideae

(33) Garcia Rohr (2 spp.)

485. G. nutans Rohr; México a Sudamérica y en las Antillas.

486. G. parviflora Lundell; endémica de México.

\section{Subfamilia Euphorbioideae}

Tribu Hippomaneae

(34) Mabea Aubl. (3 spp.)

487. M. excelsa Standl. \& Steyerm.; México a Centroamérica.

488. M. occidentalis Benth.; México a Sudamérica.

489. M. tenorioi M. Martínez Gordillo, J. Jiménez Ram. \& Cruz-Durán; endémica de México.

(35) Sebastiania Spreng. (13 spp.)

490. S. adenophora Pax \& K. Hoffm.; endémica de México.

491. S. appendiculata (Müll. Arg.) Jabl. (Sapium appendiculatum Müll. Arg.); endémica de México.

492. S. bilocularis S. Watson (Sapium biloculare (S. Watson) Pax); Megaméxico 1.

493. S. chiapensis Lundell; endémica de México.

494. S. cornuta McVaugh; endémica de México.

495. S. corniculata (Vahl) Müll. Arg.; México a Sudamérica y en las Antillas.

496. S. cruenta (Standl. \& Steyerm.) Miranda; Megaméxico 2.

497. S. hintonii Lundell; endémica de México.

498. S. longicuspis Standl.; Megaméxico 2.

499. S. lottiae McVaugh; endémica de México.

500. S. pavoniana (Müll. Arg.) Müll. Arg.; México a Centroamérica.

501. S. tikalana Lundell; Megaméxico 2.

502. S. tuerckheimiana (Pax \& K. Hoffm.) Lundell; Megaméxico 2. 
Apéndice 1. Continuación.

(36) Stillingia Gard. ex L. (12 spp.)

503. S. acutifolia (Benth.) Benth. \& Hook. f. ex Hemsl.; Megaméxico 2.

504. S. bicarpellaris S. Watson; endémica de México.

505. S. diphtherina D. J. Rogers; endémica de México.

506. S. linearifolia S. Watson; Megaméxico 1.

507. S. microsperma Pax \& K. Hoffm.; Megaméxico 2.

508. S. pietatis McVaugh; endémica de México.

509. S. querceticola McVaugh; endémica de México.

510. S. sanquinolenta Müll. Arg.; endémica de México.

511. S. spinulosa Torr.; Megaméxico 1.

512. S. texana I. M. Johnst.; Estados Unidos a México.

513. S. treculiana (Müll. Arg.) I. M. Johnst.; Megaméxico 1.

514. S. zelayensis (H.B.K.) Müll. Arg.; México a Centroamérica.

(37) Gymnanthes Sw. (4 spp.)

515. G. actinostemoides Müll. Arg.; endémica de México.

516. G. longipes Müll. Arg.; endémica de México.

517. G. lucida Sw.; Estados Unidos a Centroamérica y en las Antillas.

518. G. riparia (Schltdl.) Klotzsch; México a Centroamérica.

(38) Dalembertia Baill. (3 spp.)

519. D. platanoides Baill.; endémica de México.

520. D. populifolia Baill. (D. hahniana Baill.); endémica de México.

521. D. triangularis Müll. Arg.; Megaméxico 2.

(39) Sapium P. Browne (3 spp.)

522. S. glandulosum (L.) Morong (S. biglandulosum (L.) Müll. Arg.); México a Sudamérica y en las Antillas.

523. S. lateriflorum HemsI. (S. nitidum (Monach.) Lundell); México a Sudamérica y en las Antillas.

524. S. macrocarpum Müll. Arg. (S. mexicanum Hemsl., S. pedicellatum Huber); México a Centroamérica.

(40) Hippomane L. (1 sp.)

525. H. mancinella L.; Estados Unidos a Sudamérica y en las Antillas.

Tribu Hureae

(41) Hura L. (1 sp.)

526. $H$. polyandra Baill.; Megaméxico 2.

Tribu Euphorbieae

(42) Euphorbia L. (Chamaesyce Gray, Poinsettia Graham) (241 spp.)

527. E. abramsiana L. C. Wheeler; Megaméxico 1.

528. E. adenoptera Bertol.; Estados Unidos a México y en las Antillas.

529. E. alatocaulis V. W. Steinm. \& Felger; endémica de México.

530. E. albomarginata Torr. \& A. Gray; Estados Unidos a México.

531. E. alta Norton; Megaméxico 1.

532. E. angusta Engelm.; Megaméxico 1.

533. E. anthonyi Brandegee; endémica de México.

534. E. antisyphilitica Zucc.; Megaméxico 1.

535. E. anychioides Boiss.; Megaméxico 2.

536. E. apatzingana McVaugh; endémica de México. 
Apéndice 1. Continuación.

537. E. apicata L. C. Wheeler; endémica de México.

538. E. ariensis H.B.K.; Megaméxico 2.

539. E. arizonica Engelm.; Megaméxico 1.

540. E. armourii Millsp.; Megaméxico 2.

541. E. arteagae W. R. Buck \& Huft; endémica de México.

542. E. astyla Engelm. ex Boiss.; Megaméxico 1.

543. E. barnesii (Millsp.) Oudejans; endémica de México.

544. E. bartolomei Greene; endémica de México.

545. E. beamanii M. C. Johnst.; endémica de México.

546. E. beteroana Balb. ex Spreng.; México a Sudamérica y en las Antillas.

547. E. bifurcata Engelm.; Megaméxico 1.

548. E. bilobata Engelm.; Megaméxico 1.

549. E. blodgettii Engelm. ex Hitchc.; Estados Unidos a México y en las Antillas.

550. E. bombensis Jacq. (E. ammannioides H.B.K.); Estados Unidos a Sudamérica y en las Antillas.

551. E. brachycera Engelm.; Estados Unidos a México.

552. E. brandegeei Millsp.; endémica de México.

553. E. calcicola Fern.; endémica de México.

554. E. californica Benth.; endémica de México.

555. E. calyculata H.B.K.; endémica de México.

556. E. campestris Schltdl. \& Cham.; Megaméxico 2.

557. E. caperata McVaugh; endémica de México.

558. E. capitellata Engelm. (E. pycnanthema Engelm.); Megaméxico 1.

559. E. carunculata Waterf.; Estados Unidos a México.

560. E. ceroderma I. M. Johnst.; endémica de México.

561a. E. chaetocalyx (Boiss.) Tidestr. var. chaetocalyx; Megaméxico 1.

561b. E. chaetocalyx (Boiss.) Tidestr. var. triligulata L. C. Wheeler; Megaméxico 1.

562. E. chamberlinii I. M. Johnst.; endémica de México.

563. E. chersonesa Huft; endémica de México.

564. E. chiapensis Brandegee; Megaméxico 2.

565. E. chiribensis V. W. Steinm. \& Felger; endémica de México.

566. E. cinerascens Engelm.; Megaméxico 1.

567. E. colletioides Benth. (E. plicata S. Watson); México a Sudamérica.

568. E. colorata Engelm.; endémica de México.

569. E. cornastra (Dressler) Radcl.-Sm.; endémica de México.

570. E. correllii M. C. Johnst.; endémica de México.

571. E. cotinifolia L.; México a Sudamérica.

572. E. cozumelensis Millsp.; endémica de México.

573. E. creberrima McVaugh; endémica de México.

574a. E. crepitata L. C. Wheeler var. crepitata; endémica de México.

574b. E. crepitata L. C. Wheeler var. longa M. C. Johnst.; endémica de México.

575. E. crepuscula (L. C. Wheeler) V. W. Steinm. \& Felger; endémica de México.

576. E. cressoides M. C. Johnst.; endémica de México.

577. E. cuchumatanensis Standl. \& Steyerm.; Megaméxico 2.

578. E. cumbrae Boiss.; Megaméxico 2.

579. E. cuphosperma (Engelm.) Boiss.; Megaméxico 3.

580. E. cyathophora Murr.; México a Sudamérica e introducida en varias partes del mundo.

581. E. davidii Subils; Estados Unidos a Sudamérica e introducida en varias partes del mundo. 
Apéndice 1. Continuación.

582. E. delicatula Boiss.; endémica de México.

583. E. densiflora (Klotzsch \& Garcke) Klotzsch; México a Centroamérica.

584. E. dentata Michx.; Estados Unidos a México.

585. E. dentosa I. M. Johnst.; endémica de México.

586. E. dioeca H.B.K.; México a Sudamérica y en las Antillas.

587a. E. dioscoreoides Boiss. ssp. attenutata V. W. Steinm.; endémica de México.

587b. E. dioscoreoides Boiss. ssp. dioscoreoides; endémica de México.

588. E. eglandulosa V. W. Steinm.; endémica de México.

589. E. elata Brandegee; México a Sudamérica.

590. E. eriantha Benth.; Megaméxico 1.

591. E. exstipulata Engelm.; Megaméxico 1.

592. E. feddemae McVaugh; endémica de México.

593. E. fendleri Torr. \& A. Gray; Estados Unidos a México.

594. E. floribunda Engelm. ex Boiss.; endémica de México.

595. E. florida Engelm.; Megaméxico 1.

596. E. francoana Boiss.; Megaméxico 2.

597a. E. fruticulosa Engelm. ex Boiss. var. fruticulosa; endémica de México.

597b. E. fruticulosa Engelm. ex Boiss. var. hirtella M.C. Johnst.; endémica de México.

598. E. fulgens Karw. ex Klotzsch; endémica de México.

599a. E. furcillata H.B.K. var. furcillata; endémica de México.

599b. E. furcillata H.B.K. var. ribana M. C. Johnst.; endémica de México.

600. E. gentryi V. W. Steinm. \& T. F. Daniel; endémica de México.

601. E. georgei Oudejans (E. acuta Engelm.); Megaméxico 1.

602. E. geyeri Engelm.; Estados Unidos a México.

603. E. golondrina L. C. Wheeler; Megaméxico 1.

604. E. gracillima S. Watson; Megaméxico 1.

605. E. gradyi V. W. Steinm. \& A. Ramírez; endémica de México.

606a. E. graminea Jacq. var. graminea; México a Sudamérica.

606b. E. graminea Jacq. var. novogaliciana McVaugh (E. galiciana McVaugh); endémica de México.

607. E. grammata (McVaugh) Oudejans; endémica de México.

608. E. greggii Engelm.; endémica de México.

609. E. guadalajarana S. Watson; endémica de México.

610. E. guatemalensis Standl. \& Steyerm.; Megaméxico 2.

611. E. guiengola W. R. Buck \& Huft; endémica de México.

612. E. gumaroi Meyrán; endémica de México.

613. E. helleri Millsp.; Estados Unidos a México.

614. E. henricksonii M. C. Johnst.; endémica de México.

615. E. heterophylla L.; Estados Unidos a Sudamérica y ampliamente introducida a través del mundo.

616. E. hexagonoides S. Watson; endémica de México.

617. E. hindsiana Benth. (E. californica Benth. var. hindsiana (Benth.) Wiggins); endémica de México.

618. E. hintonii L. C. Wheeler; endémica de México.

619. E. hirsuta L. (E. pubescens Vahl); introducida de Europa.

620. E. hirta L.; Estados Unidos a Sudamérica y las Antillas y ampliamente introducida a través del mundo.

621. E. hormorhiza Radcl.-Sm.; endémica de México. 
Apéndice 1. Continuación.

622. E. humayensis Brandegee; México y de manera disyunta en Sudamérica.

623. E. hypericifolia L.; Estados Unidos a Sudamérica y ampliamente introducida a través del mundo.

624. E. hyssopifolia L.; Estados Unidos a Sudamérica.

625. E. incerta Brandegee; endémica de México.

626. E. indivisa (Engelm.) Tidestr.; Megaméxico 1.

627. E. ivanjohnstonii M. C. Johnst.; endémica de México.

628. E. ixtlana Huft; endémica de México.

629. E. jaliscensis B. L. Rob. \& Greenm.; Megaméxico 2.

630. E. johnstonii M. Mayfield; endémica de México.

631. E. lacera Boiss.; endémica de México.

632. E. lagunensis Huft; endémica de México.

633. E. lancifolia Schltdl.; Megaméxico 2.

634. E. laredana Millsp.; Megaméxico 1.

635. E. lasiocarpa Klotzsch; México a Sudamérica y en las Antillas.

636. E. lata Engelm.; Megaméxico 1.

637. E. lathyris L.; introducida de Europa.

638. E. leucantha (Klotzsch \& Garcke) Boiss.; endémica de México.

639. E. leucocephala Lotsy; Megaméxico 2.

640a. E. leucophylla Benth. ssp. comcaacorum V.W. Steinm. \& Felger; endémica de México.

640b. E. leucophylla Benth. ssp. leucophylla; endémica de México.

641. E. lineata S. Watson; endémica de México.

642. E. linguiformis McVaugh; endémica de México.

643. E. longicornuta S. Watson; endémica de México.

644. E. longicruris Scheele; Estados Unidos a México.

645. E. luciismithii B. L. Rob. \& Greenm.; endémica de México.

646. E. lundelliana Croizat; endémica de México.

647. E. lurida Engelm.; Estados Unidos a México.

648. E. macropodoides B. L. Rob. \& Greenm.; Megaméxico 2.

649. E. macropus (Klotzsch \& Garcke) Boiss. (E. biformis S. Watson); Megaméxico 3.

650. E. maculata L.; Estados Unidos a México.

651. E. macvaughii Carvajal \& J. Lomelí; endémica de México.

652. E. magdalenae Benth.; endémica de México.

653. E. marginata Pursh; Estados Unidos a México.

654. E. mcvaughiana M. C. Johnst.; endémica de México.

655. E. melanadenia Torr.; Megaméxico 1.

656. E. mendezii Boiss.; Estados Unidos a Centroamérica y en las Antillas.

657. E. mesembryanthemifolia Jacq. (E. buxifolia Lam.); Estados Unidos a Sudamérica y en las Antillas.

658. E. mexiae Standl.; endémica de México.

659. E. micromera Boiss.; Megaméxico 1.

660. E. misella S. Watson; Megaméxico 2.

661. E. misera Benth.; Megaméxico 1.

662. E. montereyana Millsp.; endémica de México.

663. E. multiseta Benth.; endémica de México.

664. E. muscicola Fernald; endémica de México.

665. E. nayarensis V. W. Steinm.; endémica de México.

666. E. neilmulleri M. C. Johnst.; endémica de México. 
Apéndice 1. Continuación.

667. E. nutans Lag.; Estados Unidos a Sudamérica e introducida en el Viejo Mundo.

668. E. oaxacana B. L. Rob. \& Greenm.; endémica de México.

669. E. ocymoidea L. (E. astroites Fisch. \& C. A. Mey.); Megaméxico 2.

670. E. oppositifolia McVaugh; endémica de México.

671. E. ophthalmica Pers. (E. hirta L. var. procumbens (Boiss.) N. E. Br.); Estados Unidos a Sudamérica.

672. E. orizabae Boiss.; Megaméxico 2.

673. E. palmeri Engelm. ex S. Watson; Estados Unidos a México.

674. E. parryi Engelm.; Estados Unidos a México.

675a. E. pediculifera Engelm. var. linearifolia S. Watson; endémica de México.

675b. E. pediculifera Engelm. var. pediculifera; Megaméxico 1.

676. E. peganoides Boiss.; endémica de México.

677. E. peninsularis I. M. Johnst.; endémica de México.

678. E. peplidion Engelm.; Estados Unidos a México.

679. E. peplus L.; introducida de Eurasia.

680. E. perennans (Shinners) Warnock \& M. C. Johnst.; Megaméxico 1.

681. E. perlignea McVaugh; endémica de México.

682. E. petrina S. Watson; endémica de México.

683. E. picachensis Brandegee; Megaméxico 2.

684. E. pinkavana M. C. Johnst.; endémica de México.

685. E. pionosperma V. W. Steinm. \& Felger; endémica de México.

686. E. platysperma Engelm. ex S. Watson; Megaméxico 1.

687a. E. polycarpa Benth. var. mejamia L. C. Wheeler; endémica de México.

687b. E. polycarpa Benth. var. polycarpa; Estados Unidos a México.

688. E. pondii Millsp.; endémica de México.

689. E. potosina Fern.; endémica de México.

690. E. prostrata Aiton; Estados Unidos a Sudamérica y en las Antillas, introducida en el Viejo Mundo.

691. E. pseudofulva Miranda; endémica de México.

692. E. pteroneura A. Berger; Megaméxico 2.

693. E. pueblensis Brandegee; endémica de México.

694. E. pulcherrima Willd. ex Klozsch; Megaméxico 2.

695. E. pumicicola Huft; endémica de México.

696a. E. radians Benth. var. radians; Megaméxico 1.

696b. E. radians Benth. var. stormiae (Croizat) Rzed. \& Calderón; endémica de México.

697. E. radioloides Boiss.; Megaméxico 2.

698. E. restiacea Benth.; endémica de México.

699. E. revoluta Engelm.; Estados Unidos a México.

700. E. rossiana Pax (E. antisyphilitica Zucc. var. luxurians Miranda); endémica de México.

701. E. rutilis (Millsp.) Standl. \& Steyerm.; endémica de México.

702. E. rzedowskii McVaugh; endémica de México.

703. E. salsuginosa McVaugh; endémica de México.

704. E. scandens H.B.K.; endémica de México.

705a. E. schlechtendalii Boiss. var. pacifica McVaugh; endémica de México.

705b. E. schlechtendalii Boiss. var. schlechtendalii; Megaméxico 2.

705c. E. schlechtendalii Boiss. var. websteri McVaugh: endémica de México.

706a. E. scopulorum Brandegee var. inornata M. C. Johnst.; endémica de México.

706b. E. scopulorum Brandegee var. nuda M. C. Johnst.; endémica de México. 
Apéndice 1. Continuación.

706c. E. scopulorum Brandegee var. scopulorum; endémica de México.

707. E. segoviensis (Klotzsch \& Garcke) Boiss.; Megaméxico 2.

708. E. serpens H.B.K.; Estados Unidos a Sudamérica y en las Antillas.

709. E. serpyllifolia Pers.; Estados Unidos a México, de manera disyunta en Sudamérica.

710. E. serrula Engelm.; Megaméxico 1.

711. E. setiloba Engelm. ex Torr.; Megaméxico 1.

712. E. simulans (L. C. Wheeler) Warnock \& M. C. Johnst.; Megaméxico 1.

713. E. sinaloensis Brandegee; endémica de México.

714. E. sloanei L. C. Wheeler; endémica de México.

715. E. sonorae Rose; endémica de México.

716. E. soobyi McVaugh; endémica de México.

717. E. spathulata Lam.; Estados Unidos a México y de manera disyunta en Sudamérica.

718. E. standleyi (Millsp.) Oudejans; endémica de México.

719. E. stictospora Engelm.; Estados Unidos a México.

720. E. strigosa Hook. \& Arn.; endémica de México.

721. E. subpeltata S. Watson; endémica de México.

722. E. subreniformis S. Watson; endémica de México.

723. E. succedanea L. C. Wheeler; endémica de México.

724. E. taluticola Wiggins; endémica de México.

725. E. tanquahuete Sessé \& Moc. (E. fulva Stapf); endémica de México.

726. E. terracina L.; introducida de Europa.

727. E. theriaca L. C. Wheeler; Megaméxico 1.

728. E. thymifolia L.; México a Sudamérica y en las Antillas, también en el Viejo Mundo.

729. E. tomentella Engelm. ex Boiss.; endémica de México.

730. E. tomentulosa S. Watson; endémica de México.

731. E. trachysperma Engelm.; Megaméxico 1.

732. E. tresmariae (Millsp.) Standl.; endémica de México.

733. E. trialata (Huft) V. W. Steinm.; endémica de México.

734. E. trichotoma H.B.K.; Estados Unidos a Centroamérica y en las Antillas.

735. E. tricolor Greenm.; endémica de México.

736. E. umbellulata Engelm. ex Boiss.; endémica de México.

737. E. aff. valis-mortae (Millsp.) J. T. Howell; Estados Unidos a México.

738. E. velleriflora (Klotzsch \& Garcke) Boiss.; Megaméxico 2.

739. E. vestita Boiss.; endémica de México.

740. E. villifera Scheele; Megaméxico 3.

741. E. violacea Greenm.; endémica de México.

742. E. whitei L. C. Wheeler; endémica de México.

743. E. wrightii Torr. \& A. Gray; Megaméxico 1.

744. E. xalapensis H.B.K.; México a Centroamérica.

745. E. xanti Engelm. ex Boiss.; endémica de México.

746. E. xbacensis Millsp.; endémica de México.

747. E. xylopoda Greenm.; endémica de México.

748. E. yucatanensis (Millsp.) Standl.; endémica de México.

749. E. zierioides Boiss.; endémica de México.

750. E. sp. (E. hirta L. var. nocens L. C. Wheeler); endémica de México.

751. E. sp. (Poinsettia schiedeana Klotzsch \& Garcke); Megaméxico 2.

752. E. sp. nov. 1; endémica de México.

753. E. sp. nov. 2; endémica de México. 
Apéndice 1. Continuación.

754. E. sp. nov. 3; endémica de México.

755. E. sp. nov. 4; endémica de México.

756. E. sp. nov. 5; endémica de México.

757. E. sp. nov. 6; endémica de México.

758. E. sp. nov. 7; endémica de México.

759. E. sp. nov. 8; endémica de México.

760. E. sp. nov. 9; endémica de México.

761. E. sp. nov. 10; endémica de México.

762. E. sp. nov. 11; endémica de México.

763. E. sp. nov. 12; endémica de México.

764. E. sp. nov. 13; endémica de México.

765. E. sp. nov. 14; endémica de México.

766. E. sp. nov. 15; Megaméxico 1.

767. E. sp. nov. 16; Megaméxico 2.

(43) Pedilanthus Neck. ex Poit. (15 spp.)

768. P. bracteatus (Jacq.) Boiss.; endémica de México.

769. P. calcaratus Schltdl.; Megaméxico 2.

770. P. coalcomanensis Croizat; endémica de México.

771. P. connatus Dressler \& Sacamano; endémica de México.

772. P. cymbiferus Schltdl.; endémica de México.

773. $P$. diazlunanus J. Lomelí \& Sahagún; endémica de México.

774. P. finkii Boiss.; endémica de México.

775. P. gracilis Dressler; endémica de México.

776. P. macrocarpus Benth.; endémica de México.

777. P. nodiflorus Millsp.; Megaméxico 2.

778. P. palmeri Millsp.; endémica de México.

779. P. pulchellus Dressler; endémica de México.

780. P. tehuacanus Brandegee; endémica de México.

781a. P. tithymaloides (L.) Poit. ssp. parasiticus (Klotzsch \& Garcke) Dressler; Megaméxico 2.

781b. P. tithymaloides (L.) Poit. ssp. tithymaloides; México a Sudamérica y en las Antillas.

782. P. tomentellus B. L. Rob. \& Greenm.; endémica de México. 